\title{
DEFORMATIONS OF THE TANGENT BUNDLE OF PROJECTIVE MANIFOLDS
}

\author{
THOMAS PETERNELL
}

\begin{abstract}
We investigate when the tangent bundle of a projective manifold has a non-trivial first order (or positive-dimensional) deformation. This leads to a new conjectural characterization of the complex projective space.
\end{abstract}

\section{Contents}

1. Introduction

2. Some general results 3

3. Surfaces 6

4. Threefolds 11

4.1. Fano threefolds 11

4.2. $\mathbb{P}_{2}$-bundles 13

4.3. $\mathbb{P}_{1}$-bundles 14

4.4. Blow-ups 16

5. Calabi-Yau threefolds: birational morphisms and flops 19

6. Higher Dimensions 31

References

\section{IntRoduction}

In the early 1990's, physicists asked to compute the dimension of the space $H^{1}\left(X, T_{X} \otimes \Omega_{X}^{1}\right)$ for a Calabi-Yau threefold $X$; see DGKM89, EH90. They computed the dimension for some special complete intersections. A few years later the problem was taken up by D.Huybrechts Huy95; he proved in particular that $H^{1}\left(X, T_{X} \otimes \Omega_{X}^{1}\right) \neq 0$ for three-dimensional Calabi-Yau complete intersections in projective spaces. In terms of deformation theory, this space parametrizes first order deformations of the holomorphic tangent bundle $T_{X}$.

In this paper we propose to systematically study deformations of the tangent bundle $T_{X}$ of any compact complex manifold $X$, up to deformations of the form $T_{X} \otimes \mathcal{L}$, where $\mathcal{L}$ is a deformation of the trivial line bundle $\mathcal{O}_{X}$. To be precise, we introduce the following notation.

1.1. Notation. Let $X$ be a compact complex manifold. We say that $T_{X}$ has a genuine first order deformation if $T_{X}$ has a deformation $\mathcal{E}$ over the double point $D$ which is not induced by a deformation of $\mathcal{O}_{X}$, i.e., $\mathcal{E} \nsucceq p^{*} T_{X} \otimes \mathcal{L}$ with $\mathcal{L}$ a deformation of $\mathcal{O}_{X}$ over $D$; here $p: X \times D \rightarrow X$ denotes the projection.

2010 Mathematics Subject Classification. 14J30, 14J32, 14J60.

Key words and phrases. tangent bundle, deformation, Calabi-Yau threefold. 
To have a genuine first oder deformation is equivalent to saying that

$$
h^{1}\left(X, T_{X} \otimes \Omega_{X}^{1}\right)>q(X):=h^{1}\left(X, \mathcal{O}_{X}\right) .
$$

Note that $T_{X}$ has a non-trivial first order deformation if and only if the morphism $\pi: \mathbb{P}\left(T_{X}\right) \rightarrow X$ has a first order deformation with fixed target $X$ which is not trivial, i.e., not constant.

In the same way we define genuine deformations of $T_{X}$ over a positive-dimensional parameter space. The obstructions to lifting a first order deformation in the sense of (1) ly in the space

$$
H^{2}\left(X, T_{X} \otimes \Omega_{X}^{1}\right) / H^{2}\left(X, \mathcal{O}_{X}\right) \simeq H^{2}\left(\mathbb{P}\left(T_{X}\right), T_{\mathbb{P}\left(T_{X}\right) / X}\right) .
$$

Thus $T_{X}$ has a non-obstructed genuine deformation provided

$$
h^{1}\left(X, T_{X} \otimes \Omega_{X}^{1}\right)-h^{2}\left(X, T_{X} \otimes \Omega_{X}^{1}\right)-q(X)+h^{2}\left(X, \mathcal{O}_{X}\right)>0 .
$$

1.2. Remark. Let $\mathcal{E} n d_{0}\left(T_{X}\right)$ denote the sheaf of traceless endomorphisms of $T_{X}$, hence

and a fortiori

$$
T_{X} \otimes \Omega_{X}^{1} \simeq \mathcal{E} n d_{0}\left(T_{X}\right) \oplus \mathcal{O}_{X}
$$

$$
\left.H^{q}\left(X, T_{X} \otimes \Omega_{X}^{1}\right)=H^{q}\left(X, \mathcal{E} n d\left(T_{X}\right)\right)=H^{q}\left(X, \mathcal{E} n d_{0}\left(T_{X}\right)\right) \oplus H^{q}\left(X, \mathcal{O}_{X}\right)\right)
$$

for all $q$. Then Equation (1.1.1) is equivalent to

$$
h^{1}\left(X, \mathcal{E} n d_{0}\left(T_{X}\right)\right) \neq 0 .
$$

whereas Equation (1.1.3) is equivalent to

$$
h^{1}\left(X, \mathcal{E} n d_{0}\left(T_{X}\right)\right)-h^{2}\left(X, \mathcal{E} n d_{0}\left(T_{X}\right)\right)>0 .
$$

The theme of this paper is now the following

1.3. Problem. Let $X$ be a projective (compact Kähler, or simply compact) manifold. Give necessary and sufficient conditions such that $T_{X}$ has a genuine first-order deformation resp. a genuine non-obstructed deformation.

In dimension 2 we show

1.4. Theorem. Let $X$ be a compact Kähler surface. Then $T_{X}$ does not have a genuine first order deformation if and only if either $X \simeq \mathbb{P}_{2}$ or if $X$ is a ball quotient $\left(\right.$ so $\left.c_{1}^{2}(X)=3 c_{2}(X)\right)$ with $H^{0}\left(X, S^{2} \Omega_{X}^{1}\right)=0$.

The case that $X$ is of general type has already been shown in CWY03. It would certainly be interesting to classify the two-dimensional ball quotients $X$ with $H^{0}\left(X, S^{2} \Omega_{X}^{1}\right)=0$.

In dimension larger than one however, things get much more complicated, even in dimension 3. Of course, the tangent bundle of the complex projective space is rigid in any dimension. In dimension 3 we prove - among other things -

1.5. Theorem. Let $X$ be a three-dimensional compact complex manifold. Then $T_{X}$ has a genuine first order deformation provided one of the following conditions is satisfied.

(1) $X$ is a Fano threefold, different from $\mathbb{P}_{3}$.

(2) Suppose $X$ is rationally connected and that $c_{1}(X)^{3} \leq 63$.

(3) $X$ is the blow-up of the smooth threefold $Y$ in a point or a smooth curve $C$. Suppose that $h^{0}\left(Y, T_{Y} \otimes \Omega_{Y}^{1}\right)=1$, e.g., $T_{Y}$ is stable for some polarization, and in case of the blow-up of $C$, additionally that $-K_{Y} \cdot C \geq 2$. 
(4) $X$ is a $\mathbb{P}_{2}$-bundle over a smooth curve.

(5) $X=\mathbb{P}(\mathcal{F})$ with a semi-stable rank two bundle over a smooth Kähler surface $S$ with $H^{1}\left(S, \mathcal{O}_{S}\right)=0$.

(6) $X$ is a Calabi-Yau threefold and $\varphi: X \rightarrow Y$ the contraction of an irreducible divisor $E$ to a point or a curve with $Y$ projective or $\varphi: X \rightarrow Y$ a small contraction, with a few exceptions, given in Theorem 5.12 and Theorem 5.16 .

It is also easy to see that the tangent bundles of smooth hypersurfaces $X \subset \mathbb{P}_{n+1}$ of degree $d \geq 2$ and dimension $n \geq 2$ have genuine first order deformations. If $X$ is a ball quotient of dimension at least three, then Siu Siu91 has shown that $T_{X}$ has no genuine deformation. Further, if $X$ is a product $X=X_{1} \times X_{2}$ with $X_{j}$ ball quotients with $q\left(X_{j}\right)=0$, then $T_{X}$ again has no genuine first order deformation. This procedure can also be iterated.

All these considerations lead to the following

1.6. Question. Suppose $X$ is a compact Kähler manifold. Then $T_{X}$ has no genuine first order deformation if and only if $X$ is one of the following.

- $X \simeq \mathbb{P}_{n}$

- $X$ is a ball quotient of dimension at least three

- $X$ is a twodimensional ball quotient such that $H^{1}\left(X, \mathcal{E} n d_{0}\left(T_{X}\right)\right)=0$

- $X$ is a product (with at least two factors)

$$
X=\Pi_{j} X_{j}
$$

with $X_{j}$ ball quotients such that $q\left(X_{j}\right)=0$ and $H^{1}\left(X_{j}, \mathcal{E} n d_{0}\left(T_{X_{j}}\right)\right)=0$ for all $j$.

Acknowledgements Many thanks go to Ulrike Peternell for carefully reading the first four parts of the paper and for suggesting many improvements. Also I would like to thank Alan Huckleberry and Stefan Nemirovski for very helpful discussions and Jun-Muk Hwang for pointing out the references CWY03 and Siu91. Finally, I would like to thank the referee for pointing out an error in section 5 and many other very valuable suggestions.

\section{Some General RESUlts}

In this section we prove some general results with a focus on blow-ups.

2.1. Proposition. Let $X$ be a compact complex manifold. Let $E \subset X$ be a smooth irreducible divisor. Assume that

(1) $H^{0}\left(E, T_{X} \mid E\right) \neq 0$.

(2) $H^{0}\left(X, \Omega_{X}^{1} \otimes T_{X}\right)=H^{0}\left(X, \Omega_{X}^{1}(\log E) \otimes T_{X}\right)$, Then $H^{1}\left(X, \Omega_{X}^{1} \otimes T_{X}\right) \neq 0$.

Proof. The residue sequence tensorized with $T_{X}$ reads

$$
0 \rightarrow \Omega_{X}^{1} \otimes T_{X} \rightarrow \Omega_{X}^{1}(\log E) \otimes T_{X} \rightarrow T_{X} \mid E \rightarrow 0 .
$$

Taking cohomology and using our assumption (2) we obtain an injection

$$
H^{0}\left(E, T_{X} \mid E\right) \rightarrow H^{1}\left(X, T_{X} \otimes \Omega_{X}^{1}\right)
$$

Hence assumption (1) gives the claim.

In the same way, we have 
2.2. Proposition. Let $X$ be a compact complex manifold. Let $E \subset X$ be a smooth irreducible divisor. Assume that

(1) $H^{0}\left(E, T_{X} \mid E\right) \neq 0$.

(2) $h^{0}\left(X, \mathcal{E} n d_{0}\left(T_{X}\right)\right)=h^{0}\left(X,\left(\Omega_{X}^{1}(\log E) \otimes T_{X}\right) / \mathcal{O}_{X}\right)$.

Then $H^{1}\left(X, \mathcal{E} n d_{0}\left(T_{X}\right) \neq 0\right.$.

Proof. Dividing by the trivial summand of $\Omega_{X}^{1} \otimes T_{X}$, the residue sequence induces an exact sequence

$$
0 \rightarrow \mathcal{E} n d_{0}\left(T_{X}\right) \rightarrow \mathcal{R}:=\left(\Omega_{X}^{1}(\log E) \otimes T_{X}\right) / \mathcal{O}_{X} \rightarrow T_{X} \mid E \rightarrow 0 .
$$

Then we conclude as before.

Since $0 \neq H^{0}\left(E, T_{E}\right) \subset H^{0}\left(E, T_{X} \mid E\right)$, we obtain

2.3. Corollary. Let $X$ be a compact complex manifold. Let $E \subset X$ be a smooth irreducible divisor. Assume that

(1) $H^{0}\left(E, T_{E}\right) \neq 0$,

(2) $H^{0}\left(X, \Omega_{X}^{1} \otimes T_{X}\right)=H^{0}\left(X, \Omega_{X}^{1}(\log E) \otimes T_{X}\right)$.

Then $H^{1}\left(X, T_{X} \otimes \Omega_{X}^{1}\right) \neq 0$.

2.4. Corollary. Let $X$ be a compact complex manifold. Let $E \subset X$ be a smooth irreducible divisor. Assume that

(1) $H^{0}\left(E, T_{E}\right) \neq 0$,

(2) $h^{0}\left(X, \mathcal{E} n d_{0}\left(T_{X}\right)\right)=h^{0}\left(X, \Omega_{X}^{1}(\log E) \otimes T_{X} / \mathcal{O}_{X}\right)$.

Then $H^{1}\left(X, \mathcal{E} n d_{0}\left(T_{X}\right)\right) \neq 0$.

2.5. Remark. The condition

$$
H^{0}\left(X, \Omega_{X}^{1} \otimes T_{X}\right)=H^{0}\left(X, \Omega_{X}^{1}(\log E) \otimes T_{X}\right),
$$

certainly holds provided

$$
\operatorname{dim} H^{0}\left(X, \Omega_{X}^{1}(\log E) \otimes T_{X}\right)=1 .
$$

2.6. Proposition. Let $X$ be a compact complex $n$-dimensional manifold, $x_{0} \in X$ and $\pi: \hat{X} \rightarrow X$ the blow up of $X$ at $x_{0}$. Then

(1) There is an exact sequence

$$
0 \rightarrow \pi_{*}\left(T_{\hat{X}} \otimes \Omega_{\hat{X}}^{1}\right) \rightarrow T_{X} \otimes \Omega_{X}^{1} \rightarrow Q \rightarrow 0
$$

with a sheaf $Q$ supported on $x_{0}$ of length $n^{2}-1$.

(2) $h^{1}\left(\hat{X}, T_{\hat{X}} \otimes \Omega_{\hat{X}}^{1}\right) \geq h^{1}\left(X, T_{X} \otimes \Omega_{X}^{1}\right)$ with strict inequality if $h^{0}\left(X, T_{X} \otimes\right.$ $\left.\Omega_{X}^{1}\right)<n^{2}$

(3) $h^{2}\left(\hat{X}, T_{\hat{X}} \otimes \Omega_{\hat{X}}^{1}\right) \leq h^{2}\left(X, T_{X} \otimes \Omega_{X}^{1}\right)$;

(4) $h^{1}\left(\hat{X}, \mathcal{E} n d_{0}\left(T_{\hat{X}}\right)\right) \geq h^{1}\left(X, \mathcal{E} n d_{0}\left(T_{X}\right)\right)$ with strict inequality if $h^{0}\left(X, T_{X} \otimes\right.$ $\left.\Omega_{X}^{1}\right)<n^{2}$

(5) $h^{2}\left(\hat{X}, \mathcal{E} n d_{0}\left(T_{\hat{X}}\right)\right) \leq h^{2}\left(X, \mathcal{E} n d_{0}\left(T_{X}\right)\right)$.

Proof. Set $E=\pi^{-1}\left(x_{0}\right)$. We clearly have an exact sequence

$$
0 \rightarrow \pi_{*}\left(T_{\hat{X}} \otimes \Omega_{\hat{X}}^{1}\right) \rightarrow T_{X} \otimes \Omega_{X}^{1} \rightarrow Q \rightarrow 0
$$

with a sheaf sheaf $Q$ which is zero outside $x_{0}$. Tenorize the exact sequence

$$
0 \rightarrow \pi^{*}\left(\Omega_{X}^{1}\right) \rightarrow \Omega_{\hat{X}}^{1} \rightarrow \Omega_{\hat{X} / X}^{1} \rightarrow 0
$$


by $T_{\hat{X}}$, take $\pi_{*}$ and use $R^{1} \pi_{*}\left(T_{\hat{X}}\right)=0$ to obtain the exact sequence

$$
0 \rightarrow \pi_{*}\left(T_{\hat{X}}\right) \otimes \Omega_{X}^{1} \rightarrow \pi_{*}\left(T_{\hat{X}} \otimes \Omega_{\hat{X}}^{1}\right) \rightarrow \pi_{*}\left(T_{\hat{X}} \mid E \otimes \Omega_{E}^{1}\right) \rightarrow 0 .
$$

Further, we have an exact sequence

$$
0 \rightarrow \pi_{*}\left(T_{\hat{X}}\right) \otimes \Omega_{X}^{1} \rightarrow T_{X} \otimes \Omega_{X}^{1} \rightarrow \mathbb{C}_{x_{0}}^{n} \otimes \Omega_{X}^{1} \rightarrow 0
$$

Then we obtain the following commutative diagram

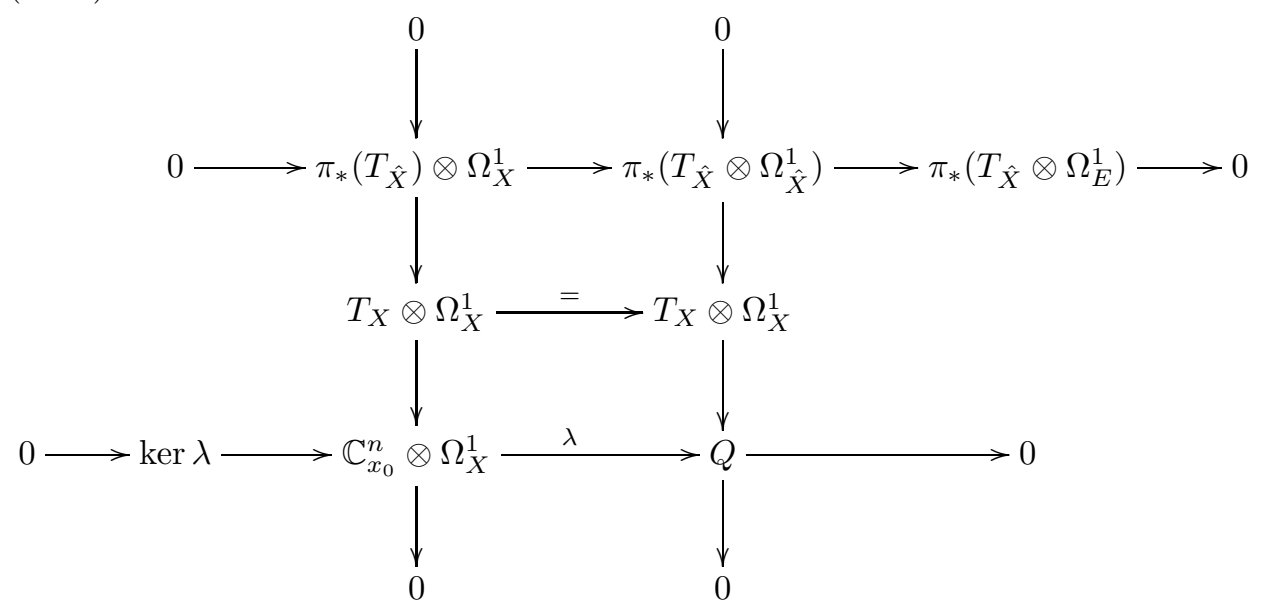

A diagram chase shows that

$$
\operatorname{ker} \lambda \simeq \pi_{*}\left(T_{\hat{X}} \mid E \otimes \Omega_{E}^{1}\right)
$$

yielding an exact sequence

$$
0 \rightarrow \pi_{*}\left(T_{\hat{X}} \mid E \otimes \Omega_{E}^{1}\right) \rightarrow \mathbb{C}_{x_{0}}^{n} \otimes \Omega_{X}^{1} \rightarrow Q \rightarrow 0 .
$$

Observe finally

$$
\pi_{*}\left(T_{\hat{X}} \mid E \otimes \Omega_{E}^{1}\right) \simeq \mathbb{C}_{x_{0}} .
$$

In fact, use the exact sequence

$$
0 \rightarrow T_{E} \otimes \Omega_{E}^{1} \rightarrow T_{\hat{X}} \mid E \otimes \Omega_{E}^{1} \rightarrow N_{E} \otimes \Omega_{E}^{1} \rightarrow 0
$$

to see that

and

$$
h^{0}\left(E, T_{\hat{X}} \mid E \otimes \Omega_{E}^{1}\right)=1
$$

$$
H^{0}\left(E, N_{E}^{* \otimes \mu} \otimes T_{\hat{X}} \mid E \otimes \Omega_{E}^{1}\right)=0
$$

for all $\mu \geq 1$, which yields (2.6.3). Then Assertion (1) follows by (2.6.3).

Assertions (2) and (3) then follow immediately by taking cohomology and using the Leray spectral sequence. Finally, (4) and (5) are immediate from (2) and (3), since $H^{q}\left(\hat{X}, \mathcal{O}_{\hat{X}}\right)=H^{q}\left(X, \mathcal{O}_{X}\right)$ for all $q$.

2.7. Corollary. Let $X$ be a compact complex $n$-dimensional manifold, $x_{0} \in X$ and $\pi: \hat{X} \rightarrow X$ the blow up of $X$ at $x_{0}$. Suppose that $T_{X}$ has a genuine first order (resp. genuine non-obstructed) deformation. Suppose further that $h^{0}\left(X, T_{X} \otimes \Omega_{X}^{1}\right)<n^{2}$. Then $T_{\hat{X}}$ has a genuine first order (genuine non-obstructed) deformation. 
If $T_{X}$ is simple, we can say more:

2.8. Theorem. Let $X$ be a compact complex manifold such that

$$
H^{0}\left(X, \Omega_{X}^{1} \otimes T_{X}\right) \simeq \mathbb{C}
$$

e.g., $T_{X}$ is stable with respect to a Gauduchon metric. Let either $\pi: \hat{X} \rightarrow X$ be the blow-up of a point in $X$ or $\operatorname{dim} X \geq 3$ and $\pi: \hat{X} \rightarrow X$ be the blow-up of a smooth rational curve $C \subset X$. Then

$$
H^{1}\left(\hat{X}, \mathcal{E} n d_{0}\left(T_{\hat{X}}\right)\right) \neq 0 .
$$

Proof. We restrict ourselves to the case of the blow-up of a curve; the point blow-up being completely analogous. We verify the conditions of Corollary 2.4. applied to the exceptional divisor $E=\pi^{-1}(C)$ and use again the notation

$$
\mathcal{R}=\left(\Omega_{\hat{X}}^{1}(\log E) \otimes T_{\hat{X}}\right) / \mathcal{O}_{\hat{X}} .
$$

Since $E=\mathbb{P}\left(N_{C / X}^{*}\right)$, the relative Euler sequence and the splitting of $N_{C / X}$ yields

$$
h^{0}\left(E, T_{E / C}\right)=h^{0}\left(C, N_{C / X}^{*} \otimes N_{C / X}\right)-1 \geq 1,
$$

thus $H^{0}\left(E, T_{E}\right) \neq 0$. As to the second condition, we first observe the following chain of inclusions and equations

$$
\begin{gathered}
H^{0}(\hat{X} \backslash E, \mathcal{R})=H^{0}\left(\hat{X} \backslash E, \mathcal{E} n d_{0}\left(T_{\hat{X})}\right)=H^{0}\left(X \backslash C, \mathcal{E} n d_{0}\left(T_{X}\right)\right)=\right. \\
=H^{0}\left(X, \mathcal{E} n d_{0}\left(T_{X}\right)\right),
\end{gathered}
$$

the last equation coming from Riemann's extension theorem.

By our assumption, $H^{0}\left(X, \mathcal{E} n d_{0}\left(T_{X}\right)\right)=0$, hence $H^{0}(\hat{X} \backslash E, \mathcal{R})=0$. Now $\mathcal{R}$ is torsion free; in fact, otherwise $\mathcal{O}_{X}$ would not be saturated in $\Omega_{X}^{1}(\log E) \otimes T_{X}$, thus $\mathcal{O}_{X} \rightarrow \Omega_{X}^{1}(\log E) \otimes T_{X}$ would vanish along $E$, which is clearly not the case. Consequently, $H^{0}(X, \mathcal{R}) \rightarrow H^{0}(X \backslash E, \mathcal{R})$ is injective, hence we conclude.

2.9. Corollary. Theorem 2.8 remains true for curves $C$ of genus $g \geq 1$, provided $H^{0}\left(T_{E}\right) \neq 0$. If $g \geq 2$, this is equivalent to

$$
h^{0}\left(N_{C} \otimes N_{C}^{*}\right) \geq 2
$$

i.e., $N_{C}$ is not simple. In case $g=1$, we might also have $h^{0}\left(N_{C} \otimes N_{C}^{*}\right)=1$ and the vector field on $C$ lifts to $E$.

2.10. Remark. Instead of assuming $T_{X}$ to be simple in Theorem 2.8, it suffices to assume that

$$
H^{0}\left(\hat{X}, T_{\hat{X}} \otimes \Omega_{\hat{X}}^{1}\right)=H^{0}\left(X, T_{X} \otimes \Omega_{X}^{1}\right) .
$$

\section{Surfaces}

We start by some general calculations.

3.1. Proposition. Let $X$ be a smooth compact complex surface. Then

(1) $\chi\left(X, T_{X} \otimes \Omega_{X}^{1}\right)=\frac{1}{3}\left(4 c_{1}^{2}(X)-11 c_{2}(X)\right)$

(2) $h^{1}\left(X, T_{X} \otimes \Omega_{X}^{1}\right)=$ $\frac{1}{3}\left(-4 c_{1}^{2}(X)+11 c_{2}(X)\right)+h^{0}\left(X, T_{X} \otimes \Omega_{X}^{1}\right)+h^{0}\left(X,\left(\Omega_{X}^{1}\right)^{\otimes 2}\right)$

(3) $h^{1}\left(X, T_{X} \otimes \Omega_{X}^{1}\right)=$ $\frac{5}{4}\left(3 c_{2}(X)-c_{1}^{2}(X)\right)+q(X)+h^{0}\left(X, \mathcal{E} n d_{0}\left(T_{X}\right)\right)+h^{0}\left(X, S^{2} \Omega_{X}^{1}\right)$. 
(4) $h^{1}\left(X, \mathcal{E} n d_{0}\left(T_{X}\right)\right)-h^{2}\left(X, \mathcal{E} n d_{0}\left(T_{X}\right)\right)=$ $\frac{5}{4}\left(3 c_{2}(X)-c_{1}^{2}(X)\right)+h^{0}\left(X, T_{X} \otimes \Omega_{X}^{1}\right)-1$.

(5) If $c_{1}^{2}(X)<3 c_{2}(X)$, then $h^{1}\left(X, \mathcal{E} n d_{0}\left(T_{X}\right)\right)-h^{2}\left(X, \mathcal{E} n d_{0}\left(T_{X}\right)\right)>0$.

Proof. (1) follows from Riemann-Roch, since $c_{1}\left(T_{X} \otimes \Omega_{X}^{1}\right)=0$ and $c_{2}\left(T_{X} \otimes \Omega_{X}^{1}\right)=$ $4 c_{2}(X)-c_{1}^{2}(X)$.

(2) is a consequence of (1), using

$$
H^{2}\left(X, T_{X} \otimes \Omega_{X}^{1}\right) \simeq H^{0}\left(X, T_{X} \otimes \Omega_{X}^{1} \otimes K_{X}\right)=H^{0}\left(X,\left(\Omega_{X}^{1}\right)^{\otimes 2}\right) .
$$

For (3), we apply (2), observe that

$$
\left(\Omega_{X}^{1}\right)^{\otimes 2} \simeq S^{2} \Omega_{X}^{1} \oplus K_{X}
$$

and use

$h^{0}\left(X, K_{X}\right)=h^{2}\left(X, \mathcal{O}_{X}\right)=\chi\left(X, \mathcal{O}_{X}\right)-1+q(X)=\frac{1}{12}\left(c_{1}^{2}(X)+c_{2}(X)\right)-1+q(X)$.

As to (4), we have, using (1),

$$
\begin{aligned}
h^{1}\left(X, \operatorname{End}_{0}\left(T_{X}\right)\right) & -h^{2}\left(X, \operatorname{End}_{0}\left(T_{X}\right)\right)=h^{1}\left(X, T_{X} \otimes \Omega_{X}^{1}\right)-h^{2}\left(X, T_{X} \otimes \Omega_{X}^{1}\right)-q(X)+ \\
+h^{2}\left(X, \mathcal{O}_{X}\right) & =-\chi\left(X, T_{X} \otimes \Omega_{X}^{1}\right)+h^{0}\left(X, T_{X} \otimes \Omega_{X}^{1}\right)+\chi\left(X, \mathcal{O}_{X}\right)-1= \\
& =\frac{45}{12} c_{2}(X)-\frac{15}{12} c_{1}^{2}(X)+h^{0}\left(X, T_{X} \otimes \Omega_{X}^{1}\right)-1 .
\end{aligned}
$$

This yields claim (4), and (5) follows from (4).

3.2. Corollary. Let $X$ be a compact complex surface. If $c_{1}^{2}(X)<3 c_{2}(X)$, then $T_{X}$ has a genuine non-obstructed deformation.

3.3. Theorem. Let $X$ be a compact complex Kähler surface. Then the following assertions are equivalent.

(1) $T_{X}$ has a genuine first order deformation.

(2) $X \not \mathbb{P}_{2}$ and $X$ is not a ball quotient with $H^{0}\left(X, S^{2} \Omega_{X}^{1}\right)=0$.

As already mentioned, surfaces of general type have been treated in CWY03.

Proof. First that if $X=\mathbb{P}_{2}$ or if $X$ is a ball quotient with $H^{0}\left(X, S^{2} \Omega_{X}^{1}\right)=0$, then by Proposition 3.1, $T_{X}$ has no genuine first order deformations. Hence Assertion (1) implies Assertion (2).

To prove the converse, we note first that by Proposition 2.6. we may assume $X$ to be minimal and by Corollary 3.2 that $c_{1}^{2}(X) \geq 3 c_{2}(X)$. The Miyaoka-Yau inequality and surface classification gives $c_{1}^{2}(X)=3 c_{2}(X)$, unless $X$ is a ruled surface over a curve of genus at least two. More specifically, again by classification, $X$ is one of the following

(1) $X=\mathbb{P}_{2}$;

(2) $X$ is a ball quotient;

(3) $\kappa(X)=1$ and $c_{2}(X)=0$;

(4) $X$ is a torus or hyperelliptic;

(5) $X$ is a ruled surface over a curve $B$ of genus $g=g(B) \geq 1$. 
Case (4) is immediately settled by Proposition 3.1(3).

(3) Assume that $\kappa(X)=1$ and $c_{2}(X)=0$. Let $f: X \rightarrow B$ be the Iitaka fibration. Suppose $T_{X}$ does not have a genuine first order deformation, then we have $h^{1}\left(X, T_{X} \otimes \Omega_{X}^{1}\right)=q(X)$. Hence $H^{0}\left(X, S^{2} \Omega_{X}^{1}\right)=0$ by Proposition 3.1(3). Hence $q(X)=0$, so that $\chi\left(X, \mathcal{O}_{X}\right)>0$, contradicting $c_{1}^{2}(X)=c_{2}(X)=0$.

(5) Let $\pi: X \rightarrow B$ denote a ruling over the curve $B$. Since $\chi\left(X, T_{X} \otimes \Omega_{X}^{1}\right)=4(g-1)$, we may assume $g \geq 2$. By Proposition 3.1 (2),

$$
\begin{gathered}
h^{1}\left(X, T_{X} \otimes \Omega_{X}^{1}\right)=4(1-g)+h^{0}\left(X, T_{X} \otimes \Omega_{X}^{1}\right)+h^{0}\left(X,\left(\Omega_{X}^{1}\right)^{\otimes 2}\right) \geq \\
\geq 4(1-g)+h^{0}\left(X, T_{X} \otimes \Omega_{X}^{1}\right)+h^{0}\left(B, 2 K_{B}\right)=1-g+h^{0}\left(X, T_{X} \otimes \Omega_{X}^{1}\right) .
\end{gathered}
$$

We will now prove that

$$
h^{0}\left(X, T_{X} \otimes \Omega_{X}^{1}\right)>3(g-1) .
$$

This yields

$$
h^{1}\left(X, T_{X} \otimes \Omega_{X}^{1}\right)>q(X)=g,
$$

which was to be proved. In order to show (3.3.1), we consider the subbundles

$$
T_{X} \otimes \pi^{*}\left(\Omega_{B}^{1}\right) \subset T_{X} \otimes \Omega_{X}^{1}
$$

and

$$
T_{X / B} \otimes \pi^{*}\left(\Omega_{B}^{1}\right) \subset T_{X} \otimes \pi^{*}\left(\Omega_{B}^{1}\right) .
$$

Write $X=\mathbb{P}(\mathcal{E})$ with a rank two bundle $\mathcal{E}$ on $B$. Then

$$
T_{X / B} \simeq \mathcal{O}_{\mathbb{P}(\mathcal{E})}(2) \otimes \pi^{*}\left(\operatorname{det} \mathcal{E}^{*}\right) .
$$

Hence

$$
\begin{gathered}
h^{0}\left(T_{X / B} \otimes \pi^{*}\left(\Omega_{B}^{1}\right)\right)=h^{0}\left(B, S^{2} \mathcal{E} \otimes \operatorname{det} \mathcal{E}^{*} \otimes \Omega_{B}^{1}\right) \geq \\
\chi\left(S^{2} \mathcal{E} \otimes \operatorname{det} \mathcal{E}^{*} \otimes \Omega_{B}^{1}\right)=3(g-1) .
\end{gathered}
$$

The last equation is Riemann-Roch for the rank three bundle $S^{2}(\mathcal{E}) \otimes \operatorname{det} \mathcal{E}^{*}$, recalling that $c_{1}\left(S^{2}(\mathcal{E}) \otimes \operatorname{det} \mathcal{E}^{*}\right)=0$. Thus

$$
h^{0}\left(X, T_{X} \otimes \Omega_{X}^{1}\right)>h^{0}\left(T_{X} \otimes \pi^{*}\left(\Omega_{B}^{1}\right)\right) \geq h^{0}\left(T_{X / B} \otimes \pi^{*}\left(\Omega_{B}^{1}\right)\right) \geq 3(g-1),
$$

proving (3.3.1). The strictness of the first equality comes from the fact that the identity map in $H^{0}\left(X, T_{X} \otimes \Omega_{X}^{1}\right)$ is not induced by an element of $H^{0}\left(X, T_{X / B} \otimes\right.$ $\pi^{*}\left(\Omega_{B}^{1}\right)$.

In summary, if $T_{X}$ does not have a genuine deformation, then $X$ is either $\mathbb{P}_{2}$ or a ball quotient, and in the latter case, necessarily $H^{0}\left(X, S^{2} \Omega_{X}^{1}\right)=0$ by Proposition 3.1(3).

3.4. Remark. There are non-Kähler surfaces whose tangent bundles have no genuine first order deformations. For example, let $X$ be an Inoue surface of type $S_{N}^{+}$, Ino74. These are exactly the surfaces with $\kappa(X)=-\infty$, having no curves and $c_{1}^{2}(X)=c_{2}(X)=0$. Moreover

$$
h^{0}\left(X, T_{X}\right)=h^{1}\left(X, T_{X}\right)=1,
$$

[no74, Prop.3]. Let $v \in H^{0}\left(X, T_{X}\right)$ be a non-zero vector field. Then $v$ has no zeroes and induces an exact sequence

$$
0 \rightarrow \mathcal{O}_{X} \rightarrow T_{X} \rightarrow \mathcal{O}_{X}\left(-K_{X}\right) \rightarrow 0 .
$$


From Riemann-Roch, we have $\chi\left(\Omega_{X}^{1}\right)=0$, hence $H^{1}\left(X, \Omega_{X}^{1}\right)$ (since $H^{0}\left(X, \Omega_{X}^{1}\right)=$ $\left.H^{2}\left(X, \Omega_{X}^{1}\right)=0\right)$. Thus taking cohomology of the preceeding exact sequence, tensorized by $\Omega_{X}^{1}$, we obtain

$$
0=H^{1}\left(X, \Omega_{X}^{1}\right) \rightarrow H^{1}\left(X, T_{X} \otimes \Omega_{X}^{1}\right) \rightarrow H^{1}\left(X, T_{X}\right) \simeq \mathbb{C},
$$

and therefore

$$
h^{1}\left(X, T_{X} \otimes \Omega_{X}^{1}\right)=1=h^{1}\left(X, \mathcal{O}_{X}\right) .
$$

Theorem 3.3 has the following partial strengthening

3.5. Theorem. Let $X$ be a compact Kähler surface. Assume that $X$ is neither $\mathbb{P}_{2}$, nor a ball quotient nor of the form $\mathbb{P}(\mathcal{E})$ with $\mathcal{E}$ a stable locally free sheaf of rank two over an elliptic curve. Then $T_{X}$ has a genuine non-obstructed deformation.

Proof. By Proposition 2.6, we may assume $X$ minimal. What remains to be proved is the following. Suppose $X$ is one of the following.

(1) $\kappa(X)=1$ and $c_{2}(X)=0$

(2) $X$ is a torus or hyperelliptic

(3) $X$ is a ruled surface over a curve $B$ of genus $g=g(B) \geq 1$, but not of the form $\mathbb{P}(\mathcal{E})$ with $\mathcal{E}$ a stable locally free sheaf of rank two over an elliptic curve.

Then $T_{X}$ has a non-trivial non-obstructed deformation.

(1) Assume first that $\kappa(X)=1$. By Proposition 3.1(4), it suffices to show that

$$
h^{0}\left(X, T_{X} \otimes \Omega_{X}^{1}\right) \geq 2 \text {. }
$$

To do this, consider the Iitaka fibration $f: X \rightarrow B$. Since $c_{2}(X)=0$, the only singular fiber of $f$ are multiples $m_{i} F_{i}$ of elliptic curves $F_{i}$; write $D=\sum\left(m_{i}-1\right) F_{i}$. The elliptic bundle formula now reads

$$
K_{X}=f^{*}\left(K_{B} \otimes \mathcal{L}\right) \otimes \mathcal{O}_{X}(D)
$$

with a torsion line bundle $\mathcal{L}$. Further, there is an exact sequence

$$
0 \rightarrow f^{*}\left(K_{B}\right) \otimes \mathcal{O}_{X}(D) \rightarrow \Omega_{X}^{1} \rightarrow K_{X / B} \otimes \mathcal{O}_{X}(-D) \rightarrow 0,
$$

and therefore an inclusion

$$
T_{X} \otimes f^{*} K_{B} \otimes \mathcal{O}_{X}(D) \subset T_{X} \otimes \Omega_{X}^{1} .
$$

Thus it suffices to show

$$
H^{0}\left(X, T_{X} \otimes f^{*} K_{B} \otimes \mathcal{O}_{X}(D)\right) \neq 0 .
$$

Indeed, a non-zero element in the space is a morphism $\Omega_{X}^{1} \rightarrow f^{*} K_{B} \otimes \mathcal{O}_{X}(D)$. Composing with the inclusion $f^{*} K_{B} \otimes \mathcal{O}_{X}(D) \rightarrow \Omega_{X}^{1}$ yields a morphism $\Omega_{X}^{1} \rightarrow \Omega_{X}^{1}$ which is not a multiple of id. Dualizing the last exact sequence yields an inclusion

$$
f^{*}\left(K_{B} \otimes \mathcal{L}^{*}\right) \otimes \mathcal{O}_{X}(D) \simeq K_{X / B}^{*} \otimes \mathcal{O}_{X}(2 D) \otimes f^{*}\left(K_{B}\right) \rightarrow T_{X} \otimes f^{*} K_{B} \otimes \mathcal{O}_{X}(D) .
$$

Now

$$
H^{0}\left(X, f^{*}\left(K_{B} \otimes \mathcal{L}^{*}\right) \otimes \mathcal{O}_{X}(D)\right)=H^{0}\left(B, K_{B} \otimes \mathcal{L}^{*}\right) \neq 0
$$

unless $g=1$ and $\mathcal{L}$ not trivial, we are done except for this special case. Here we perform a finite étale base change $\tilde{B} \rightarrow B$ to trivialize $\mathcal{L}$ and set $\tilde{X}=X \times_{B} \tilde{B}$ with projection $\tilde{f}: \tilde{X} \rightarrow \tilde{B}$. Then the associated line bundle $\tilde{\mathcal{L}}$ and therefore

$$
h^{0}\left(\tilde{X}, T_{\tilde{X}} \otimes \Omega_{\tilde{X}}^{1}\right) \geq 2 .
$$


Thus there exists a morphism

$$
\lambda: \Omega_{\tilde{X}}^{1} \rightarrow \Omega_{\tilde{X}}^{1},
$$

which is not a multiple of the identity. Let $\mu: \tilde{X} \rightarrow X$ be the projection and consider

$$
\mu_{*}(\lambda): \mu_{*}\left(\Omega_{\tilde{X}}^{1}\right) \rightarrow \mu_{*}\left(\Omega_{\tilde{X}}^{1}\right) .
$$

Via the decomposition

$$
\mu_{*}\left(\Omega_{\tilde{X}}^{1}\right)=\mu_{*} \mu^{*}\left(\Omega_{X}^{1}\right)=\Omega_{X}^{1} \otimes \mu_{*}\left(\mathcal{O}_{\tilde{X}}\right)=\Omega_{X}^{1} \otimes \bigoplus_{j=0}^{-s} \mathcal{L}^{j},
$$

there exists a number $k$ and a non-zero morphism

$$
\psi: \Omega_{X}^{1} \rightarrow \Omega_{X}^{1} \otimes \mathcal{L}^{k}
$$

We aim to prove that $k=0$; hence we obtain an endomorphism of $\Omega_{X}^{1}$ which is not a multiple of the identity and conclude. Using the cotangent sequence, which now reads

$$
0 \rightarrow \mathcal{O}_{X}(D) \otimes \Omega_{X}^{1} \rightarrow K_{X} \otimes \mathcal{O}_{X}(-D) \rightarrow 0,
$$

the morphism $\psi$ induces by composition a morphism

$$
\psi_{1}: \mathcal{O}_{X}(D) \rightarrow K_{X} \otimes \mathcal{O}_{X}(-D) \otimes f^{*}\left(\mathcal{L}^{k}\right)=f^{*}\left(\mathcal{L} \otimes \mathcal{L}^{k}\right) .
$$

If $\psi_{1} \neq 0$, then $k=-1$ and $D=0$, a contradiction. Therefore $\psi_{1}$ and $\psi$ induces a nonvanishing map

$$
\mathcal{O}_{X}(D) \rightarrow \mathcal{O}_{X}(D) \otimes f^{*}\left(\mathcal{L}^{k}\right)
$$

hence $k=0$.

(2) If $X$ is a torus or hyperelliptic, then

$$
h^{0}\left(X, T_{X} \otimes \Omega_{X}^{1}\right)=h^{0}\left(X, \Omega_{X}^{1} \otimes \Omega_{X}^{1}\right)=h^{0}\left(X, S^{2} \Omega_{X}^{1}\right)+h^{0}\left(X, K_{X}\right) \geq 2,
$$

hence we conclude again by Proposition $3.1(4)$.

(3) Finally, let $p: X \rightarrow B$ be a ruled surface over a curve $B$ of genus $g=g(B) \geq 1$. Consider the relative tangent bundle sequence

$$
0 \rightarrow T_{X / B} \rightarrow T_{X} \rightarrow p^{*}\left(T_{B}\right) \rightarrow 0
$$

and its associated extension class $\zeta \in H^{1}\left(X, T_{T / B} \otimes p^{*}\left(T_{B}^{*}\right)\right) \simeq H^{1}\left(X,-K_{X}\right)$. Now $H^{1}\left(X,-K_{X}\right) \neq 0$, unless $g=1$ and $X=\mathbb{P}(\mathcal{E})$ with $\mathcal{E}$ a stable locally free sheaf of rank two on $B$. This is a direct consequence of the structure results of ruled surfaces, Har77, chap. V.2]. The latter case ruled out by assumption, we can deform the extension class $\zeta$ and obtain a deformation $\mathcal{F}$ of $T_{X}$ over $X \times \Delta$. Every $\mathcal{F}_{t}$ sits in an exact sequence

$$
0 \rightarrow T_{X / B} \rightarrow \mathcal{F}_{t} \rightarrow p^{*}\left(T_{B}\right) \rightarrow p^{*}\left(T_{B}\right) \rightarrow 0 .
$$

Since there are no non-trivial maps $T_{X / B} \rightarrow p^{*}\left(T_{B}\right)$, the sheaves $\mathcal{F}_{t}$ are different from $T_{X}$ for $t \neq 0$, and we obtain a non-trivial positive-dimensional deformation of $T_{X}$.

It remains to treat the case $X=\mathbb{P}(\mathcal{E})$ with $\mathcal{E}$ stable over the elliptic curve $B$. 
3.6. Remark. Assume that $X=\mathbb{P}(\mathcal{E})$ with $\mathcal{E}$ a stable locally free sheaf of rank two over an elliptic curve or that $X$ is a ball quotient with $H^{0}\left(X, S^{2} \Omega_{X}^{1}\right) \neq 0$. Then $T_{X}$ has a genuine first order deformation and one might suspect that a suitable such deformation is not obstructed. Then Theorem 3.5 could be stated as follows: $T_{X}$ has a genuine non-obstructed deformation if and only if $X$ is neither $\mathbb{P}_{2}$ nor a ball quotient with $H^{0}\left(X, S^{2} \Omega_{X}^{1}\right)=0$.

\section{ThrEeFoldS}

The Riemann-Roch formula gives, using $c_{2}\left(T_{X} \otimes \Omega_{X}^{1}\right)=-2 c_{1}^{2}(X)+6 c_{2}(X)$,

4.1. Proposition. Let $X$ be a 3-dimensional compact complex manifold. Then

$$
\chi\left(X, T_{X} \otimes \Omega_{X}^{1}\right)=c_{1}^{3}(X)-\frac{63}{24} c_{1}(X) c_{2}(X) .
$$

\subsection{Fano threefolds.}

4.2. Proposition. Let $X$ be a Fano threefold. Then $T_{X}$ has a genuine nonobstructed deformation unless $X=\mathbb{P}_{3}$.

Proof. Since $\chi\left(X, \mathcal{O}_{X}\right)=1$, we have $c_{1}(X) c_{2}(X)=24$ by Riemann-Roch. Hence

$$
\chi\left(X, T_{X} \otimes \Omega_{X}^{1}\right)=c_{1}^{3}(X)-63 .
$$

By the classification of Fano threefolds, $c_{1}^{3}(X) \leq 62$, unless $X=\mathbb{P}_{3}$. Notice further that

$$
H^{3}\left(X, T_{X} \otimes \Omega_{X}^{1}\right)=H^{0}\left(X, \Omega_{X}^{1} \otimes \Omega_{X}^{2}\right)=0,
$$

e.g., since $X$ is rationally connected.

Hence

$$
h^{1}\left(X, T_{X} \otimes \Omega_{X}^{1}\right)-h^{2}\left(X, T_{X} \otimes \Omega_{X}^{1}\right)>0,
$$

unless $X=\mathbb{P}_{3}$. Since $q(X)=0$, this proves the claim.

The arguments actually show more (having in mind that $h^{0}\left(X, T_{X} \otimes \Omega_{X}^{1}\right) \neq 0$ )

4.3. Corollary. Let $X$ be a smooth threefold with $\chi\left(X, \mathcal{O}_{X}\right) \geq 0$ and

$$
H^{0}\left(X, \Omega_{X}^{1}\right)=H^{0}\left(X, \Omega_{X}^{2}\right)=H^{0}\left(X, \Omega_{X}^{1} \otimes \Omega_{X}^{2}\right)=0 .
$$

Assume that

$$
c_{1}^{3}(X) \leq 63 .
$$

Then $T_{X}$ has a genuine non-obstructed deformation.

If $X$ is rationally connected, then the first two conditions in Corollary 4.3 are satisified, hence we obtain

4.4. Corollary. Let $X$ be a smooth rationally connected threefold. Assume that

$$
c_{1}^{3}(X) \leq 63 .
$$

Then $T_{X}$ has a non-trivial non-obstructed deformation.

In view of Proposition 4.2 it is natural to consider the case that $X$ is "weak Fano", i.e., $-K_{X}$ is big and nef. In that case, $c_{1}^{3}(X) \leq 72$ by [Pro05, Thm.1.5]. In fact, for a suitable positive integer $m$ the line bundle $-m K_{X}$ is spanned by global sections and defines a birational morphism $\varphi: X \rightarrow Y$ to a Fano Gorenstein variety $Y$ with at most canonical singularities such that $-K_{X}=\varphi^{*}\left(-K_{Y}\right)$. By 
Pro05, Thm.1.5], $\left(-K_{Y}\right)^{3} \leq 72$, hence $c_{1}^{3}(X)=\left(-K_{X}\right)^{3} \leq 72$. The bound 72 is sharp; actually $\left(-K_{Y}\right)^{3}=72$ if and only if $Y$ is either the weighted projective space $\mathbb{P}(3,1,1,1)$ or $\mathbb{P}(6,4,1,1)$. Thus we cannot conclude directly that $T_{X}$ has a first order or non-obstructed deformation. It should however be possible to classifiy all $X$ in the range $64 \leq c_{1}^{3}(X) \leq 72$ and treat this cases by hand. We give one example, namely

$$
X=\mathbb{P}\left(\mathcal{O}_{\mathbb{P}_{2}} \oplus \mathcal{O}_{\mathbb{P}_{2}}(3)\right) .
$$

In this case $Y=\mathbb{P}(3,1,1,1)$. For simplicity, we consider only first order deformations.

4.5. Proposition. Let $X$ be the weak Fano threefold $\mathbb{P}\left(\mathcal{O}_{\mathbb{P}_{2}} \oplus \mathcal{O}_{\mathbb{P}_{2}}(3)\right)$. Then $T_{X}$ has a genuine first order deformation.

We prepare the proof by the following

4.6. Lemma. Let $\pi: X \rightarrow S$ be a $\mathbb{P}_{1}$-bundle over the smooth compact surface $S$. Assume that $h^{0}\left(S, T_{S} \otimes \Omega_{S}^{1}\right)=1$. Then

$$
h^{0}\left(X, T_{X} \otimes \Omega_{X}^{1}\right)=1+h^{0}\left(X, T_{X / S} \otimes \Omega_{X}^{1}\right)=1+h^{0}\left(X,-K_{X / S} \otimes \Omega_{X}^{1}\right) .
$$

Proof. The sequence

$$
0 \rightarrow T_{X / S} \otimes \Omega_{X}^{1} \rightarrow T_{X} \otimes \Omega_{X}^{1} \rightarrow \pi^{*}\left(T_{S}\right) \otimes \Omega_{X}^{1} \rightarrow 0
$$

yields in cohomology

$$
0 \rightarrow H^{0}\left(X, T_{X / S} \otimes \Omega_{X}^{1}\right) \rightarrow H^{0}\left(X, T_{X} \otimes \Omega_{X}^{1}\right) \stackrel{\alpha}{\rightarrow} H^{0}\left(X, \pi^{*}\left(T_{S}\right) \otimes \Omega_{X}^{1}\right) .
$$

The sequence

$$
0 \rightarrow \pi^{*}\left(T_{S}\right) \otimes \pi^{*}\left(\Omega_{S}^{1}\right) \rightarrow \pi^{*}\left(T_{S}\right) \otimes \Omega_{X}^{1} \rightarrow \pi^{*}\left(T_{S}\right) \otimes K_{X / S} \rightarrow 0
$$

shows

$$
H^{0}\left(X, \pi^{*}\left(T_{S}\right) \otimes \Omega_{X}^{1}\right)=H^{0}\left(S, T_{S} \otimes \Omega_{S}^{1}\right) \simeq \mathbb{C} .
$$

Hence it suffices to observe that $\alpha \neq 0$. This is however clear: id $: \Omega_{X}^{1} \rightarrow \Omega_{X}^{1}$ yields via $\alpha$ a non-zero morphism $\pi^{*}\left(\Omega_{S}^{1}\right) \rightarrow \Omega_{X}^{1}$.

Proof of Proposition 4.5 By Proposition 2.1, applied to the exceptional section $E:=\mathbb{P}\left(\mathcal{O}_{\mathbb{P}_{2}}\right) \simeq \mathbb{P}_{2}$ in $X$, it suffices to show that

$$
H^{0}\left(X, T_{X} \otimes \Omega_{X}^{1}\right)=H^{0}\left(X, T_{X} \otimes \Omega_{X}^{1}(\log E)\right) .
$$

We use the exact sequence

$$
0 \rightarrow T_{X / S} \otimes \Omega_{X}^{1}(\log E) \rightarrow T_{X} \otimes \Omega_{X}^{1}(\log E) \rightarrow \pi^{*}\left(T_{S}\right) \otimes \Omega_{X}^{1}(\log E) \rightarrow 0 .
$$

Using Lemma 4.6, things come down to show

$$
H^{0}\left(X, T_{X / S} \otimes \Omega_{X}^{1}\right)=H^{0}\left(X, T_{X / S} \otimes \Omega_{X}^{1}(\log E)\right)
$$

and

$$
h^{0}\left(X, \pi^{*}\left(T_{S}\right) \otimes \Omega_{X}^{1}(\log E)\right)=1 .
$$

The first equation is seen by taking cohomology of the exact sequence

$$
0 \rightarrow T_{X / S} \otimes \Omega_{X}^{1} \rightarrow T_{X / S} \otimes \Omega_{X}^{1}(\log E) \rightarrow T_{X / S} \mid E \rightarrow 0
$$

and by observing

$$
H^{0}\left(E, T_{X / S} \mid E\right)=H^{0}\left(E,-K_{X / S} \mid E\right)=H^{0}\left(E, N_{E / X}\right)=0 .
$$


The second equation follows from the observation

$$
\pi_{*}\left(\Omega_{X}^{1}\right)=\pi_{*}\left(\Omega_{X}^{1}(\log E)\right),
$$

which is seen either by restricting to the fibers of $\pi$ or by noticing that, taking $\pi_{*}$, the induced morphism $\mathcal{O}_{S} \rightarrow R^{1} \pi_{*}\left(\Omega_{X}^{1}\right)$ is injective. Thus Equation (4.6.1) is shown and the proof of Proposition 4.5 is complete.

In Subsection 4.3 we come back to $\mathbb{P}_{1}$-bundles over surfaces in general. How the later results do not yield Proposition 4.5 .

4.2. $\mathbb{P}_{2}$-bundles. We start to study threefolds carrying a projective bundle structure by studying $\mathbb{P}_{2}$-bundles.

4.7. Theorem. Let $\pi: X \rightarrow C$ be a $\mathbb{P}_{2}$-bundle over the smooth projective curve $C$. Then $T_{X}$ has a genuine first order deformation.

Proof. Write $X=\mathbb{P}(\mathcal{F})$ with a locally free sheaf $\mathcal{F}$ of rank three on $C$. Let $g$ be the genus of $C$. If $g=0$, then $\left(-K_{X}\right)^{3}=54$, hence we conclude by Corollary 4.4 , To compute $\left(-K_{X}\right)^{3}$, just use the formula

$$
\left.-K_{X}=\mathcal{O}_{\mathbb{P}(\mathcal{F})}(3) \otimes \pi^{*}\left(\operatorname{det} \mathcal{F}^{*}\right) \otimes \mathcal{O}_{C}\left(-K_{C}\right)\right),
$$

see e.g. [Har77, Ex.III.8.4].

Thus we will assume from now on that $g \geq 1$. In this case $\chi\left(X, \mathcal{E} n d_{0}\left(T_{X}\right)\right) \geq 0$, so a more detailled investigation has to be made. Taking cohomology of the exact sequence

$$
0 \rightarrow T_{X / C} \otimes \Omega_{X}^{1} \rightarrow T_{X} \otimes \Omega_{X}^{1} \rightarrow \pi^{*}\left(T_{C}\right) \otimes \Omega_{X}^{1} \rightarrow 0
$$

and observing that the morphism

$$
H^{0}\left(X, T_{X} \otimes \Omega_{X}^{1}\right) \rightarrow H^{0}\left(X, \pi^{*}\left(T_{C}\right) \otimes \Omega_{X}^{1}\right)=H^{0}\left(X, \pi^{*}\left(T_{C} \otimes \Omega_{C}^{1}\right)\right) \simeq \mathbb{C}
$$

is surjective, leads to an exact sequence

$$
\begin{aligned}
0 \rightarrow H^{1}\left(X, T_{X / C} \otimes \Omega_{X}^{1}\right) & \rightarrow H^{1}\left(X, T_{X} \otimes \Omega_{X}^{1}\right) \rightarrow H^{1}\left(X, \pi^{*}\left(T_{C}\right) \otimes \Omega_{X}^{1}\right) \rightarrow \\
& \rightarrow H^{2}\left(X, T_{X / C} \otimes \Omega_{X}^{1}\right) .
\end{aligned}
$$

We will now show

(1) $h^{1}\left(X, T_{X / C} \otimes \Omega_{X}^{1}\right) \geq g$;

(2) $H^{1}\left(X, \pi^{*}\left(T_{C}\right) \otimes \Omega_{X}^{1}\right) \neq 0$;

(3) $H^{2}\left(X, T_{X / C} \otimes \Omega_{X}^{1}\right)=0$.

Then the exact sequence yields

$$
h^{1}\left(X, T_{X} \otimes \Omega_{X}^{1}\right)>g=h^{1}\left(X, \mathcal{O}_{X}\right),
$$

which was to be proved.

Proof of (1). Write $\mathcal{L}:=\mathcal{O}_{\mathbb{P}(\mathcal{F})}(1)$ and tensor the relative Euler sequence

$$
0 \rightarrow \mathcal{O}_{X} \rightarrow \pi^{*}\left(\mathcal{F}^{*}\right) \otimes \mathcal{L} \rightarrow T_{X / C} \rightarrow 0
$$

by $\Omega_{X}^{1}$ to obtain an exact sequence

$$
H^{1}\left(X, T_{X / C} \otimes \Omega_{X}^{1}\right) \rightarrow H^{2}\left(X, \Omega_{X}^{1}\right) \rightarrow H^{2}\left(X, \pi^{*}\left(\mathcal{F}^{*}\right) \otimes \mathcal{L} \otimes \Omega_{X}^{1}\right) .
$$

Now $H^{2}\left(X, \pi^{*}\left(\mathcal{F}^{*}\right) \otimes \mathcal{L} \otimes \Omega_{X}^{1}\right)=0$ via the Leray spectral sequence. Further $h^{2}\left(X, \Omega_{X}^{1}\right)=g>0$ : use Hodge decomposition and $H^{3,0}=0$ to obtain

$$
h^{2}\left(X, \Omega_{X}^{1}\right)=\frac{b_{3}(X)}{2}=\frac{b_{1}(C)}{2}=g(C) .
$$


Hence (1) follows.

Proof of (2). Since $\pi_{*}\left(\Omega_{X}^{1}\right)=\Omega_{C}^{1}$, we have

$$
h^{1}\left(X, \pi^{*}\left(T_{C}\right) \otimes \Omega_{X}^{1}\right) \geq h^{1}\left(C, T_{C} \otimes \Omega_{C}^{1}\right)=g,
$$

proving (2).

Proof of (3). This follows again by the Leray spectral sequence.

4.3. $\mathbb{P}_{1}$-bundles. In many cases the non-rigidity of the tangent bundle of a $\mathbb{P}_{1}$ bundle over a surface $S$ can be established as follows. For simplicity, we assume that $q(X)=H^{1}\left(X, \mathcal{O}_{X}\right)=H^{1}\left(S, \mathcal{O}_{S}\right)=0$.

4.8. Proposition. Let $\pi: X \rightarrow S$ be a $\mathbb{P}_{1}$-bundle over the smooth compact surface $S$ with $q(S)=0$. If

$$
H^{1}\left(X, T_{X} \otimes \pi^{*}\left(\Omega_{S}^{1}\right)\right)=H^{1}\left(S, \pi_{*}\left(T_{X}\right) \otimes \Omega_{S}^{1}\right) \neq 0,
$$

then $T_{X}$ has a genuine first order deformation.

Proof. We use the exact sequence

$$
0 \rightarrow T_{X} \otimes \pi^{*}\left(\Omega_{S}^{1}\right) \rightarrow T_{X} \otimes \Omega_{X}^{1} \rightarrow T_{X} \otimes K_{X / S} \rightarrow 0 .
$$

From the exact sequence

$$
0 \rightarrow \mathcal{O}_{X}=-K_{X / S} \otimes K_{X / S} \rightarrow T_{X} \otimes K_{X / S} \rightarrow \pi^{*}\left(T_{S}\right) \otimes K_{X / S} \rightarrow 0,
$$

we deduce $h^{0}\left(X, T_{X} \otimes K_{X / S}\right)=1$. Now

$$
H^{0}\left(X, T_{X} \otimes \Omega_{X}^{1}\right) \rightarrow H^{0}\left(X, T_{X} \otimes K_{X / S}\right)
$$

does not vanish: id : $T_{X} \rightarrow T_{X}$ induces a non-zero morphism $-K_{X / S} \rightarrow T_{X}$. Hence by Sequence (4.8.1),

$$
H^{1}\left(X, T_{X} \otimes \pi^{*}\left(\Omega_{S}^{1}\right)=H^{1}\left(S, \pi_{*}\left(T_{X}\right) \otimes \Omega_{S}^{1}\right)\right.
$$

injects into $H^{1}\left(X, T_{X} \otimes \Omega_{X}^{1}\right)$.

4.9. Corollary. Let $\pi: X \rightarrow S$ be a $\mathbb{P}_{1}$-bundle over the smooth compact surface $S$ with $q(S)=0$. If

$$
h^{0}\left(S, T_{S} \otimes \Omega_{S}^{1}\right)=1
$$

and if

$$
h^{1}\left(X, T_{X / S} \otimes \pi^{*}\left(\Omega_{S}^{1}\right)\right) \geq 2,
$$

then $T_{X}$ has a genuine first order deformation.

Proof. This is immediate, taking cohomology of

$$
0 \rightarrow T_{X / S} \otimes \pi^{*}\left(\Omega_{S}^{1}\right) \rightarrow T_{X} \otimes \pi^{*}\left(\Omega_{S}^{1}\right) \rightarrow \pi^{*}\left(T_{S} \otimes \Omega_{S}^{1}\right) \rightarrow 0 .
$$

With a little more care and a slighty stronger assumption on $h^{1}\left(X, T_{X / S} \otimes\right.$ $\left.\pi^{*}\left(\Omega_{S}^{1}\right)\right)$, but without assumption on $q(S)$, we obtain non-obstructed deformations: 
4.10. Proposition. Let $\pi: X \rightarrow S$ be a $\mathbb{P}_{1}$-bundle. Assume that

$$
h^{0}\left(S, T_{S} \otimes \Omega_{S}^{1}\right)=1
$$

and that

$$
h^{1}\left(X, T_{X / S} \otimes \pi^{*}\left(\Omega_{S}^{1}\right)\right) \geq 3 .
$$

Then $T_{X}$ has a genuine non-obstructed deformation.

Proof. We consider the tangent bundle sequence

$$
0 \rightarrow T_{X / S} \longrightarrow T_{X} \stackrel{\alpha}{\longrightarrow} \pi^{*}\left(T_{S}\right) \rightarrow 0 .
$$

Since

$$
\operatorname{dim} \operatorname{Ext}^{1}\left(\pi^{*}\left(T_{S}\right), T_{X / S}\right)=h^{1}\left(X, T_{X / S} \otimes \pi^{*}\left(\Omega_{S}^{1}\right)\right) \geq 3,
$$

we obtain an at least three-dimensional family of extensions

$$
0 \rightarrow T_{X / S} \stackrel{\beta}{\longrightarrow} \mathcal{E}_{t} \longrightarrow \pi^{*}\left(T_{S}\right) \rightarrow 0,
$$

and it suffices to show that $\mathcal{E}_{t} \neq T_{X}$ for general $t$. Assume to the contrary that $\mathcal{E}_{t} \simeq T_{X}$. Then the composed map $\alpha \circ \beta: T_{X / S} \rightarrow \pi^{*}\left(T_{S}\right)$ must vanish (restrict to fibers of $\pi$ ). Hence $\beta$ induces a morphism $T_{X / S} \rightarrow T_{X / S}$ which must be a multiple of the identity map. Thus we have an induced map $\pi^{*}\left(T_{S}\right) \rightarrow \pi^{*}\left(T_{S}\right)$, which by assumption is another multiple of the identity. Hence the space of extension is twodimensional, contradicting our dimension assumption.

We now give a criterion for the nonvanishing of $H^{1}\left(X, T_{X / S} \otimes \pi^{*}\left(\Omega_{S}^{1}\right)\right)$. We assume for simplicity that the $\mathbb{P}_{1}$-bundle $X \rightarrow S$ is actually of the form $X=\mathbb{P}(\mathcal{F})$ with a locally free sheaf $\mathcal{F}$ of rank two on $S$; this can always arranged by passing to a finite étale cover of $S$, see Ele82.

4.11. Corollary. Let $\mathcal{F}$ be a locally free sheaf of rank two on the smooth compact complex surface $S$ with $q(S)=0$ and set $X=\mathbb{P}(\mathcal{F})$. Assume that

$$
h^{0}\left(S, T_{S} \otimes \Omega_{S}^{1}\right)=1
$$

and that

$$
2 c_{1}^{2}(\mathcal{F})-8 c_{2}(\mathcal{F})+\frac{1}{2}\left(K_{S}^{2}-5 c_{2}(S)\right) \leq-2 .
$$

Then $T_{X}$ has a genuine first order deformation. This happens e.g., when $\mathcal{F}$ is $\omega$-semistable for some Kähler form $\omega$, so that $c_{1}^{2}(\mathcal{F}) \leq 4 c_{2}(\mathcal{F})$.

Proof. Notice that

$$
\pi_{*}\left(T_{X / S}\right)=S^{2}(\mathcal{F}) \otimes \operatorname{det} \mathcal{F}^{*},
$$

thus

$$
h^{1}\left(S, S^{2}(\mathcal{F}) \otimes \operatorname{det} \mathcal{F}^{*} \otimes \Omega_{S}^{1}\right)=h^{1}\left(X, T_{X / S} \otimes \pi^{*}\left(\Omega_{S}^{1}\right) .\right.
$$

Hence it suffices to show that

$$
\chi\left(S, S^{2}(\mathcal{F}) \otimes \operatorname{det} \mathcal{F}^{*} \otimes \Omega_{S}^{1}\right) \leq-2,
$$

which is equivalent by Riemann-Roch to our assumption.

Notice finally that since $q(S)=0$, then $K_{S}^{2} \leq 3 c_{2}(S)$ and $c_{2}(S)=\chi_{\text {top }}(S) \geq 3$.

In the same manner, we have 
4.12. Corollary. Let $\mathcal{F}$ be a locally free sheaf of rank two on the smooth compact complex surface $S$ and set $X=\mathbb{P}(\mathcal{F})$. Assume that $h^{0}\left(S, T_{S} \otimes \Omega_{S}^{1}\right)=1$ and that

$$
2 c_{1}^{2}(\mathcal{F})-8 c_{2}(\mathcal{F})+\frac{1}{2}\left(K_{S}^{2}-5 c_{2}(S)\right) \leq-3 .
$$

Then $T_{X}$ has a genuine unobstructed deformation.

4.13. Remark. The equation $H^{1}\left(X, T_{X} \otimes \pi^{*}\left(\Omega_{S}^{1}\right)\right) \neq 0$ also holds under the assumptions

$$
H^{1}\left(S, T_{S} \otimes \Omega_{S}^{1}\right) \neq 0
$$

and

$$
h^{0}\left(S, S^{2}\left(\mathcal{F}^{*}\right) \otimes \operatorname{det} \mathcal{F} \otimes \Omega_{S}^{1}\right)=0,
$$

again by taking cohomology of the exact sequence

$$
\left.0 \rightarrow T_{X / S} \otimes \pi^{*}\left(\Omega^{1}\right) S\right) \rightarrow T_{X} \otimes \pi^{*}\left(\Omega_{S}^{1}\right) \rightarrow \pi^{*}\left(T_{S} \otimes \Omega_{S}^{1}\right) \rightarrow 0 .
$$

4.14. Remark. In our setting $X=\mathbb{P}(\mathcal{F})$, we have

$$
\chi\left(X, \mathcal{E} n d_{0}\left(T_{X}\right)\right)=2 c_{1}^{2}(\mathcal{F})-8 c_{2}(\mathcal{F})+\frac{2}{3}\left(K_{S}^{2}-8 c_{2}(S)\right) .
$$

So if this number is negative and if

$$
\left.H^{3}\left(X, \mathcal{E} n d_{0}\left(T_{X}\right)\right)\right)=0,
$$

then $H^{1}\left(X, \mathcal{E} n d_{0}\left(T_{X}\right)\right) \neq 0$. The last vanishing amounts via Serre duality to

$$
\left.h^{0}\left(X, \Omega_{X}^{1} \otimes \Omega_{X}^{2}\right) \leq h^{0}\left(X, K_{X}\right)\right) .
$$

4.4. Blow-ups. We conclude the section by studying blow-ups. First we recollect our knowledge on blow-ups of points.

4.15. Proposition. Let $Y$ be a smooth compact complex threefold and $\pi: X \rightarrow Y$ be the blow-up at $y_{0}$ with exceptional divisor $E$. Then $T_{X}$ has a genuine first order deformation provided one of the following holds.

(1) $Y$ is rationally connected with $c_{1}^{3}(Y) \leq 71$;

(2) $Y$ is smooth threefold with $c_{1}^{3}(Y) \leq 71$ and $Y$ is uniruled.

If the $M R C$ fibration has two-dimensional (smooth) image $S$, suppose further that $\chi\left(S, \mathcal{O}_{S}\right) \neq 0$ and that $H^{0}\left(S, \Omega_{S}^{1} \otimes K_{S}\right)=0$;

(3) $T_{Y}$ has a genuine first order deformation and $h^{0}\left(Y, T_{Y} \otimes \Omega_{Y}^{1}\right)<9$;

(4) $h^{0}\left(Y, T_{Y} \otimes \Omega_{Y}^{1}\right)=1$, i.e., $T_{Y}$ is simple.

In the cases (1) and (2) $T_{X}$ has even a genuine non-obstructed deformation; the same being true in case (3) provided $T_{Y}$ has a non-obstructed genuine deformation.

Proof. Assertion (1) is settled by Corollary 4.4. (3) by Corollary 2.7 and (4) by Theorem 2.8. Thus only (2) needs to be proven. We calculate

$$
\begin{gathered}
\chi\left(X, \mathcal{E} n d_{0}\left(T_{X}\right)\right)=\chi\left(X, T_{X} \otimes \Omega_{X}^{1}\right)-\chi\left(X, \mathcal{O}_{X}\right)= \\
=c_{1}^{3}(X)-64 \chi\left(X, \mathcal{O}_{X}\right)=c_{1}^{3}(X)-64 \chi\left(S, \mathcal{O}_{S}\right) \leq 63-64 \chi\left(S, \mathcal{O}_{S}\right) .
\end{gathered}
$$

Hence by our assumption $\chi\left(S, \mathcal{O}_{S}\right) \geq 1$ and therefore $\chi\left(X, \mathcal{E} n d_{0}\left(T_{X}\right)\right)<0$ and therefore

$$
h^{1}\left(X, \mathcal{E} n d_{0}\left(T_{X}\right)\right)-h^{2}\left(X, \mathcal{E} n d_{0}\left(T_{X}\right)\right)+h^{3}\left(X, \mathcal{E} n d_{0}\left(T_{X}\right)\right)>0 .
$$

Hence it suffices to show that

$$
H^{3}\left(X, T_{X} \otimes \Omega_{X}^{1}\right)=0 .
$$


By Serre duality,

$$
H^{3}\left(X, T_{X} \otimes \Omega_{X}^{1}\right)=H^{0}\left(X, T_{X} \otimes \Omega_{X}^{1} \otimes \mathcal{O}_{X}\left(K_{X}\right)\right)=H^{0}\left(X, \Omega_{X}^{1} \otimes \Omega_{X}^{2}\right) .
$$

We may assume that $Y$ is not rationally connected. $Y$ being uniruled, we consider the MRC fibration $f: X \rightarrow S$, Kol96, with $S$ not uniruled and smooth. If $\operatorname{dim} S=2$, Proposition 4.17 shows that

$$
H^{0}\left(X, \Omega_{X}^{1} \otimes \Omega_{X}^{2}\right)=H^{0}\left(S, \Omega_{S}^{1} \otimes K_{S}\right) .
$$

If $\operatorname{dim} S=1$, then $S$ is a smooth curve of genus at least two, and $f$ is actually a morphism. But then, restricting to a general fiber $F$, a rational surface, a direct calculation shows that

$$
H^{0}\left(X, \Omega_{X}^{1} \otimes \Omega_{X}^{2}\right)=0
$$

proving (4.15.1).

In the proof of the next proposition we will use the following

4.16. Notation. Let $X$ be a normal complex algebraic variety. Then

$$
\Omega_{X}^{[q]}:=\left(\bigwedge^{q} \Omega_{X}^{1}\right)^{* *}
$$

denotes the sheaf of reflexive $q$-forms. If $X$ has canonical singularites and if $\pi$ : $\hat{X} \rightarrow X$ is a desingularization, then by [GKKP11],

$$
\Omega_{X}^{[q]}=\pi_{*}\left(\Omega_{\hat{X}}^{q}\right) .
$$

4.17. Proposition. Let $X$ be a smooth projective threefold. Suppose that $X$ is uniruled with $M R C$ fibration $f: X \rightarrow S$ to the smooth projective surface $S$. Then the pull-back

$$
f^{*}: H^{0}\left(S, \Omega_{S}^{1} \otimes K_{S}\right) \rightarrow H^{0}\left(X, \Omega_{X}^{1} \otimes \Omega_{X}^{2}\right)
$$

is an isomorphism.

Proof. Note that $\operatorname{dim} h^{0}\left(X, \Omega_{X}^{1} \otimes \Omega_{X}^{2}\right)$ is a birational invariant of smooth projective manifolds. Hence we may assume that $f$ is a morphism. Clearly, $f^{*}$ is injective. Running a relative MMP, we obtain a factorization

$$
X \rightarrow X^{\prime} \rightarrow S,
$$

where $X \rightarrow X^{\prime}$ is a sequence of relative contractions and flips, and where $f^{\prime}$ : $X^{\prime} \rightarrow S$ is a Mori fiber space.

We proceed with the following observations. If $Y$ and $Z$ are normal projective varieties with terminal singularities and if $\varphi: Y \rightarrow Z$ is a divisorial contraction, then

$$
\varphi_{*}\left(\left(\Omega_{Y}^{1} \otimes \Omega_{Y}^{2}\right)^{* *}\right) \subset\left(\Omega_{Z}^{1} \otimes \Omega_{Z}^{2}\right)^{* *} .
$$

Moreover, if $\varphi: Y \rightarrow Z$ is a flip, then

$$
H^{0}\left(Y,\left(\Omega_{Y}^{1} \otimes \Omega_{Y}^{2}\right)^{* *}\right)=H^{0}\left(Z,\left(\Omega_{Z}^{1} \otimes \Omega_{Z}^{2}\right)^{* *}\right) .
$$

Hence it suffices to consider $f^{\prime}: X^{\prime} \rightarrow S$, which is equidimensional and a conic bundle outside a finite set of $S$, i.e., there is a finite set $A \subset S$ such that $f^{\prime}$ : $X^{\prime} \backslash\left(f^{\prime}\right)^{-1}(A) \rightarrow S \backslash A$ is a conic bundle, and we need to prove that

$$
\left(f^{\prime}\right)^{*}: H^{0}\left(S, \Omega_{S}^{1} \otimes \Omega_{S}^{2}\right) \rightarrow H^{0}\left(X^{\prime},\left(\Omega_{X^{\prime}}^{1} \otimes \Omega_{X^{\prime}}^{2}\right)^{* *}\right)
$$


is surjective. Since $f^{\prime}$ is a submersion outside a set of dimension at most one, $\Omega_{X^{\prime} / S}^{1}$ is torsion free. We will use the exact sequences

$$
0 \rightarrow f^{\prime *}\left(\Omega_{S}^{1}\right) \rightarrow \Omega_{X^{\prime}}^{1} \rightarrow \Omega_{X^{\prime} / S}^{1} \rightarrow 0
$$

and

$$
0 \rightarrow f^{\prime *}\left(\Omega_{S}^{2}\right) \rightarrow \Omega_{X^{\prime}}^{[2]} \rightarrow \mathcal{S} \rightarrow 0
$$

where $\mathcal{S}$ is a torsion free sheaf with $\mathcal{S}\left|X_{0}^{\prime}=\left(f^{\prime *}\left(\Omega_{S}^{1}\right) \otimes \Omega_{X^{\prime} / S}^{1}\right)\right| X_{0}^{\prime}$ and where $X_{0}^{\prime}$ is the regular locus of $X^{\prime}$ and $f^{\prime}$. Now we observe that

$$
H^{0}\left(X^{\prime},\left(\Omega_{X^{\prime} / S}^{1} \otimes \Omega_{X^{\prime}}^{[2]}\right)^{* *}\right)=0
$$

and

$$
H^{0}\left(X^{\prime}, \mathcal{S} \otimes\left(f^{\prime}\right)^{*}\left(\Omega_{S}^{1}\right)\right)=0 .
$$

Indeed, both sheaves in question are negative on the general fiber of $f^{\prime}$. Then the assertion follows, tensoring the first exact sequence with $\Omega_{X^{\prime}}^{[2]}$, and then the second with $\left(f^{\prime}\right)^{*}\left(\Omega_{S}^{1}\right)$ and computing on $X_{0}^{\prime}$.

Proposition 4.15] suggests to proceed by induction on the Picard number $\rho(X)$, performing an MMP. In this context, we notice that in a similar way as in Proposition 4.15, it possible to treat the other contraction of extremal rays on threefolds which contract a divisor $E$ to a point. This opens a way to reduce the problem to Mori fiber spaces and threefolds with nef canonical bundles (possibly singular).

Blowing up curves is more complicated; we restrict ourselves to first order deformations.

4.18. Theorem. Let $Y$ be a smooth projective threefold such that

$$
h^{0}\left(Y, T_{Y} \otimes \Omega_{Y}^{1}\right)=1 .
$$

Let $\pi: X \rightarrow Y$ be the blow-up of a smooth curve $C \subset Y$. Assume that

$$
-K_{Y} \cdot C \geq 2 .
$$

Then $H^{1}\left(X, \mathcal{E} n d_{0}\left(T_{X}\right)\right) \neq 0$.

Proof. It suffices to show that

$$
H^{1}\left(Y, \pi_{*}\left(\mathcal{E} n d_{0}\left(T_{X}\right)\right) \neq 0 .\right.
$$

To prove this, we consider the canonical exact sequence

$$
0 \rightarrow \pi_{*}\left(\mathcal{E} n d_{0}\left(T_{X}\right)\right) \rightarrow \mathcal{E} n d_{0}\left(T_{Y}\right) \rightarrow Q \rightarrow 0,
$$

where $Q$ is a coherent sheaf supported on $C$. Then things comes down to show that

$$
H^{0}(Y, Q) \neq 0 \text {. }
$$

The sheaf $Q$ appears as well in the exact sequence

$$
0 \rightarrow \pi_{*}\left(T_{X} \otimes \Omega_{X}^{1}\right) \rightarrow T_{Y} \otimes \Omega_{Y}^{1} \rightarrow Q \rightarrow 0
$$

and we shall work with this sequence. Set $E=\pi^{-1}(C)$. The normal bundle of $C$ in $Y$ will simply be denoted $N_{C}$. Using the exact sequences

$$
0 \rightarrow \pi_{*}\left(T_{X}\right) \otimes \Omega_{Y}^{1} \rightarrow \pi_{*}\left(T_{X} \otimes \Omega_{X}^{1}\right) \rightarrow \pi_{*}\left(T_{X} \mid E \otimes \Omega_{E / C}^{1}\right) \rightarrow 0
$$

and

$$
0 \rightarrow \pi_{*}\left(T_{X}\right) \otimes \Omega_{Y}^{1} \rightarrow T_{Y} \otimes \Omega_{Y}^{1} \rightarrow N_{C} \otimes \Omega_{Y}^{1} \rightarrow 0,
$$


a diagram chase yields an exact sequence

$$
0 \rightarrow \pi_{*}\left(T_{X} \mid E \otimes \Omega_{E / C}^{1}\right) \rightarrow N_{C} \otimes \Omega_{Y}^{1} \mid C \rightarrow Q \rightarrow 0 .
$$

Since

$$
\begin{gathered}
h^{0}\left(C, \pi_{*}\left(T_{X} \mid E \otimes \Omega_{E / C}^{1}\right)\right)=h^{0}\left(E, T_{X} \mid E \otimes \Omega_{E / C}^{1}\right)= \\
=h^{0}\left(E, T_{E} \otimes \Omega_{E / C}^{1}\right)=h^{0}\left(E, T_{E / C} \otimes \Omega_{E / C}^{1}\right)=h^{0}\left(E, \mathcal{O}_{E}\right)=1,
\end{gathered}
$$

Equation (4.18.2) comes therefore down to show that

$$
h^{0}\left(C, N_{C} \otimes \Omega_{Y}^{1} \mid C\right) \geq 2 .
$$

This follows the stronger inequality

$$
\chi\left(C, N_{C} \otimes \Omega_{Y}^{1} \mid C\right) \geq 2,
$$

which by Riemann-Roch is equivalent to

$$
6(1-g)+c_{1}\left(N_{C} \otimes \Omega_{Y}^{1} \mid C\right) \geq 2 .
$$

This is just our assumption via the adjunction formula.

4.19. Corollary. Let $Y$ be a Fano threefold such that $h^{0}\left(Y, T_{Y} \otimes \Omega_{Y}^{1}\right)=1$. Let $\pi: X \rightarrow Y$ be the blow-up of a smooth curve $C \subset Y$. Then

$$
H^{1}\left(X, \mathcal{E} n d_{0}\left(T_{X}\right)\right) \neq 0 .
$$

Proof. It remains to treat the case that $-K_{Y} \cdot C=1$. But then $C$ is a smooth rational curve, and Theorem 2.8 applies. In fact, if $-K_{Y} \cdot C=1$, then $Y$ must have index one. In almost all cases, $-K_{Y}$ is spanned, hence defines a morphism $\varphi: Y \rightarrow \mathbb{P}_{N}$ such that $\varphi^{*}\left(\mathcal{O}_{\mathbb{P}_{N}}\right)(1)=\mathcal{O}_{Y}\left(-K_{Y}\right)$. Hence $\varphi(C)$ must be a line $\ell$ and $C \rightarrow \ell$ is an isomorphism. There are only two exceptional cases, [IP99, p.49] which can be checked by hand.

Keeping track of the $H^{1}$-term in $\chi\left(N_{C} \otimes \Omega_{Y}^{1} \mid C\right)$ and using Serre duality, the proof of Theorem 4.18 actually shows the slightly stronger

4.20. Corollary. Let $Y$ be a smooth projective threefold such that

$$
h^{0}\left(Y, T_{Y} \otimes \Omega_{Y}^{1}\right)=1 .
$$

Let $\pi: X \rightarrow Y$ be the blow-up of a smooth curve $C \subset Y$. If

$$
-K_{Y} \cdot C+h^{0}\left(N_{C} \otimes \Omega_{Y}^{2} \mid C\right) \geq 2,
$$

then $H^{1}\left(X, \mathcal{E} n d_{0}\left(T_{X}\right)\right) \neq 0$.

Proof. It suffices to note that in case $g=0$, Theorem 2.8 applies.

\section{Calabi-Yau threefolds: Birational morphisms and flops}

We are now turning to Calabi-Yau manifolds, mostly in dimension three. To be precise, a Calabi-Yau manifold is a simply connected projective manifold $X$ with $K_{X} \simeq \mathcal{O}_{X}$. Therefore $T_{X}$ has a genuine first order deformation if and only if $H^{1}\left(X, T_{X} \otimes \Omega_{X}^{1}\right) \neq 0$. 
5.1. Definition. Let $X$ be a Calabi-Yau manifold and $\varphi: X \rightarrow Y$ be a birational morphism to a normal projective variety $Y$. Then $\varphi$ is said to be primitive if the relative Picard number $\rho(X / Y)=1$. A primitive contraction is divisorial if the exceptional locus $E$ of $\varphi$ has codimension 1. Then automatically $E$ is an irreducible divisor.

We first collect a few known results on divisorial contractions in dimension three.

5.2. Proposition. Let $X$ be a Calabi-Yau threefold, $\varphi: X \rightarrow Y$ a primitive divisorial birational map contracting the irreducible divisor $E$. Then the following holds.

(1) $K_{Y} \simeq \mathcal{O}_{Y}$ and $Y$ has canonical singularities; we say that $Y$ is a weak Calabi-Yau variety.

(2) If $\operatorname{dim} \varphi(E)=0$, then $-K_{E}$ is ample, so $E$ is a (possibly singular) del Pezzo surface.

(3) If $\operatorname{dim} \varphi(E)=1$, then $C:=\varphi(E)$ is a smooth curve. The map $\varphi_{\mid E}$ defines a conic bundle structure on $E$.

Proof. We refer to the fundamental papers of Wilson Wil92, Wil93, Wil94, Wil97, Wil99.

A primitive contraction might also be a small birational contraction, in which case the exceptional locus is a finite union of smooth rational curves. This case will be treated at the end of this section. Or we have $\operatorname{dim} Y=1$ or 2 ; then $\varphi$ is a K3-fibration, an abelian fibration or an elliptic fibration. These cases will be treated in a different paper. Note also that it is expected that a Calabi-Yau threefold $X$ with $\rho(X) \geq 2$ should always admit a (non-trivial) contraction. This follows from two standard conjectures, namely that the Mori cone $\overline{N E}(X)$ is locally rational polyhedral and that nef line bundles on Calabi-Yau manifolds should be semiample; see e.g. the above cited papers of Wilson or the survey [LOP18.

Recall that, given be a normal complex algebraic variety $X$, then

$$
\Omega_{X}^{[q]}:=\left(\bigwedge^{q} \Omega_{X}^{1}\right)^{* *}
$$

is the sheaf of reflexive $q$-forms. Further, if $X$ has canonical singularites and if $\pi: \hat{X} \rightarrow X$ is a desingularization, then by GKKP11,

$$
\Omega_{X}^{[q]}=\pi_{*}\left(\Omega_{\hat{X}}^{q}\right) .
$$

5.3. Lemma. Let $X$ be a Calabi-Yau manifold, $\varphi: X \rightarrow Y$ a primitive birational morphism to a normal projective variety, contracting an irreducible divisor $E$. Then the following holds.

(1) The reflexive cotangent sheaf $\Omega_{Y}^{[1]}$ is $H$-polystable for any ample divisor $H$ on $X$.

(2) If $\Omega_{Y}^{[1]}$ is not $H$-stable for some ample divisor $H$, then there exists a quasiétale cover $\eta: Z \rightarrow Y$ such that $Z$ decomposes into a product an abelian variety and (possibly singular) Calabi-Yau varieties and irreducible symplectic varieties (in the sense of GKP16) (possibly $Z$ is just an abelian variety or there is no abelian factor).

(3) Assume $n=3$ and that $\Omega_{Y}^{[1]}$ is not $H$-stable for some ample divisor $H$. Then $\operatorname{dim} \varphi(E)=1$ and there exists a quasi-étale cover $\eta: Z \rightarrow Y$ such 
that either $Z$ is an abelian threefold or $Z=B \times S$ with $Z$ an elliptic curve and $S$ a K3-surface (possibly with rational double points).

Proof. Assertion (1) has been shown by Guenancia [Gue16, while Assertion (2) is the main result in [HP19. Note that either $Z$ is abelian or $Z$ has a product decomposition with at least two factor, since Calabi-Yau or irreducible symplectic varieties have stable tangent sheaves (even after quasi-étale cover).

So it remains to prove (3). By (2), it is clear that $Z$ is either abelian or a product $B \times S$ with $B$ an elliptic curve and $S$ a K3-surface. So suppose that $\operatorname{dim} \varphi(E)=0$, i.e., $Y$ has a single singular points $y_{0}$, a case which we will rule out. Note that $Z$ can have at most finitely many singular point, hence if $Z=C \times S$, hence $S$ is smooth. Thus $Z$ always smooth. We consider the orbifold Euler characteristic $e_{\text {orb }}(Y)$, see e.g. [Bla96, 2.14]. By [Bla96, Cor. p.26],

$$
e_{\mathrm{orb}}(Z)=\operatorname{deg}(\eta) \cdot e_{\mathrm{orb}}(Y) \text {. }
$$

Now $e_{\text {orb }}(Z)=e_{\text {top }}(Z)=0$. On the other hand,

$$
e_{\mathrm{orb}}(Y)=e_{\mathrm{top}}(Y)-\left(1-\frac{1}{\text { ordG }_{\mathrm{y}_{0}}}\right),
$$

where $G_{y_{0}}$ is the group attached to the quotient singularity $y_{0}$, which is absurd since $e_{\text {top }}(Y)$ is an integer.

The case $n=3$ and $E$ is contracted to a curve is more complicated; here we simply state what is needed later.

5.4. Lemma. Let $X$ be a Calabi-Yau threefold, $\varphi: X \rightarrow Y$ a primitive birational morphism to a normal projective variety, contracting an irreducible divisor $E$ to the smooth curve $C$. If $C \simeq \mathbb{P}_{1}$ (or, more generally, the genus $g(C)$ is even) and if $E$ is a $\mathbb{P}_{1}$-bundle over $C$, then $\Omega_{Y}^{[1]}$ is $H$-stable for any ample divisor $H$.

Proof. We argue as in the previous proof, part (3). Still we have $e_{\mathrm{orb}}(C \times S)=0$, even if $S$ is singular. This is immediate from the definition of the orbifold Euler number, since $C$ is a elliptic curve. Since $E$ is a $\mathbb{P}_{1}$-bundle, $Y$ is locally of the form $\Delta \times\left(A_{1}\right)$ with $\Delta$ a small disc in $\mathbb{C}$. Thus for all $y \in C$, the group $G_{y}$ has order 2 and by definition of the orbifold Euler number,

$$
e_{\text {orb }}(Y)=e_{\text {top }}(Y)-\left(1-\frac{1}{2} e_{\text {top }}(C)\right) \text {. }
$$

Since $C \simeq \mathbb{P}_{1}$ by assumption, we conclude that

$$
e_{\text {top }}(Y)=1 \text {, }
$$

hence $b_{3}(Y)$ is odd. This is impossible: either use the Leray spectral sequence to deduce that $H^{3}(X, \mathbb{C})=H^{3}(Y, \mathbb{C})$. But $b_{3}(X)$ is even due to Hodge decomposition on $X$.

5.5. Remark. If $C$ is an elliptic curve in the setting of Lemma 5.4 then we conclude that $\chi_{\text {top }}(Y)=0$, hence $\chi_{\text {top }}(X)=4$.

5.6. Proposition. Let $X$ be a Calabi-Yau manifold, $\varphi: X \rightarrow Y$ a primitive divisorial birational map contracting the irreducible divisor E. Suppose that $\Omega_{Y}^{[1]}$ is $H$-stable for some ample divisor $H$. Then

$$
\operatorname{dim} H^{0}\left(X, \Omega_{X}^{1} \otimes T_{X}\right)=\operatorname{dim} H^{0}\left(X, \mathcal{O}_{X}(E) \otimes \Omega_{X}^{1} \otimes T_{X}\right)=1 .
$$


Proof. The equation $\operatorname{dim} H^{0}\left(X, \Omega_{X}^{1} \otimes T_{X}\right)=1$ follows from the stability of $T_{X}$. To obtain the second equation, observe that any non-zero section of $\Omega_{X}^{1}(E) \otimes T_{X}$ can be seen as a non-zero morphism

$$
\lambda: \Omega_{X}^{1}(-E) \rightarrow \Omega_{X}^{1} .
$$

Taking direct images, this gives a morphism

$$
\varphi_{*}(\lambda): \varphi_{*}\left(\Omega_{X}^{1}(-E)\right) \rightarrow \varphi_{*}\left(\Omega_{X}^{1}\right) .
$$

Since $\varphi_{*}\left(\Omega_{X}^{1}(-E)\right) \subset \Omega_{Y}^{[1]}$, with equality outside $S=\varphi(E)$, the map $\varphi_{*}(\lambda)$ gives a morphism

$$
\mu^{\prime}:\left(\Omega_{Y}^{[1]}\right)_{\mid Y \backslash S} \rightarrow\left(\Omega_{Y}^{[1]}\right)_{\mid Y \backslash S} .
$$

Since $S$ has codimension at least 2 in $Y$, and since $\Omega_{Y}^{[1]}$ is reflexive, $\mu^{\prime}$ extends to a morphism

$$
\mu: \Omega_{Y}^{[1]} \rightarrow \Omega_{Y}^{[1]} .
$$

By construction, $\varphi_{*}(\lambda)=\mu \circ \iota$, where

$$
\iota: \varphi_{*}\left(\Omega_{X}^{1}(-E)\right) \rightarrow \Omega_{Y}^{[1]}
$$

is the inclusion map. Now $\Omega_{Y}^{[1]}$ is stable for any ample line bundle on $Y$ by Lemma 5.3. In particular, $\Omega_{Y}^{[1]}$ is simple, thus there exists a complex number $c \neq 0$ such that

$$
\mu=c \text { id. }
$$

It follows that $\lambda_{\mid X \backslash E}=c \operatorname{id}_{\Omega_{X \backslash E}^{1}}$, and therefore $\lambda=c \operatorname{id}_{\Omega_{X}^{1}} \circ \kappa$, where $\kappa: \Omega_{X}^{1}(-E) \rightarrow$ $\Omega_{X}^{1}$ is the inclusion map. This proves the assertion.

5.7. Lemma. With $n=\operatorname{dim} X$, suppose in the setting of Proposition 5.6 additionally that $H^{n-1}\left(E, \Omega_{X}^{1} \otimes T_{X} \mid E\right) \neq 0$. Then

$$
H^{1}\left(X, T_{X} \otimes \Omega_{X}^{1}\right) \neq 0 .
$$

Proof. We show equivalently that $H^{n-1}\left(X, \Omega_{X}^{1} \otimes T_{X}\right) \neq 0$. Consider the cohomology sequence

$$
\begin{gathered}
H^{n-1}\left(X, \Omega_{X}^{1} \otimes T_{X}\right) \rightarrow H^{n-1}\left(E, \Omega_{X}^{1} \otimes T_{X} \mid E\right) \rightarrow \\
\rightarrow H^{n}\left(X, \mathcal{O}_{X}(-E) \otimes \Omega_{X}^{1} \otimes T_{X}\right) \rightarrow H^{n}\left(X, \Omega_{X}^{1} \otimes T_{X}\right) \rightarrow 0 .
\end{gathered}
$$

From Proposition 5.6 and Serre duality, we know that

$$
\operatorname{dim} H^{n}\left(X, \mathcal{O}_{X}(-E) \otimes \Omega_{X}^{1} \otimes T_{X}\right)=\operatorname{dim} H^{n}\left(X, \Omega_{X}^{1} \otimes T_{X}\right) .
$$

This yields the claim.

5.8. Theorem. Let $X$ be a Calabi-Yau manifold, $\varphi: X \rightarrow Y$ a primitive divisorial birational map contracting the irreducible divisor $E$. If $H^{0}\left(E, T_{E}\right) \neq 0$, and if $\Omega_{Y}^{[1]}$ is $H$-stable for some ample divisor $H$, then

$$
H^{1}\left(X, \Omega_{X}^{1} \otimes T_{X}\right) \neq 0 .
$$


Proof. We aim to apply Proposition 5.7 and verify that

$$
\left.H^{n-1}\left(E, \Omega_{X}^{1} \otimes T_{X}\right) \mid E\right)=H^{0}\left(E, T_{X} \otimes \Omega_{X}^{1} \otimes K_{E}\right) \neq 0 .
$$

In fact, $T_{X} \otimes \Omega_{X}^{1} \mid E \otimes K_{E}$ contains - via the (co)tangent sequence and the adjunction formula - the subsheaf

$$
T_{E} \otimes N_{E}^{*} \otimes K_{E} \simeq T_{E}
$$

hence the nonvanishing follows from our assumption $H^{0}\left(E, T_{E}\right) \neq 0$.

In case $E$ is smooth, Theorem 5.8 also follows from Corollary 2.3 .

5.9. Remark. Suppose that $\operatorname{dim} X=3$. The condition $H^{0}\left(E, T_{E}\right) \neq 0$ holds in the following cases $E$ smooth).

(1) $E$ be a del Pezzo surface and $K_{E}^{2} \geq 6$.

(2) $E$ is a rational ruled surface.

(3) $E$ is a ruled surface over an elliptic curve, and the vector field on the elliptic curve lifts to $E$.

(4) $E$ is a ruled surface over $C$, and $E=\mathbb{P}(V)$ with a rank 2-vector bundle $V$ such that $h^{0}\left(V^{*} \otimes V\right) \geq 2$, since by the relative Euler sequence, the relative vector fields are computed by

$$
h^{0}\left(E, T_{E / C}\right)=h^{0}\left(C, V^{*} \otimes V\right)-1
$$

If $H^{0}\left(E, T_{E}\right)=0$, things get more involved, we restrict ourselves to dimension three. The key is the following

5.10. Proposition. Let $X$ be a Calabi-Yau threefold, $\varphi: X \rightarrow Y$ be a birational morphism to a normal compact complex (Moishezon) space $Y$, whose exceptional locus is a smooth rational curve $C$. Then $H^{1}\left(X, T_{X} \otimes \Omega_{X}^{1}\right) \neq 0$.

Proof. We argue by contradiction and assume to the contrary that

$$
H^{1}\left(X, T_{X} \otimes \Omega_{X}^{1}\right)=0 .
$$

By a theorem of Laufer [Lau81, Thm.4.1], the normal bundle $N_{C}=N_{C / X}$ has the following form

(1) $N_{C}=\mathcal{O}_{C}(-1) \oplus \mathcal{O}_{C}(-1)$;

(2) $N_{C}=\mathcal{O}_{C} \oplus \mathcal{O}_{C}(-2)$;

(3) $N_{C}=\mathcal{O}_{C}(1) \oplus \mathcal{O}_{C}(-3)$.

Moreover, $y_{0}=\varphi(C)$ is a hypersurface singularity.

We claim that

$$
h^{0}\left(Y, R^{1} \varphi_{*}\left(T_{X} \otimes \Omega_{X}^{1}\right)\right) \geq 5 .
$$

To prove Claim (5.10.1), we use the inequality

$$
h^{0}\left(Y, R^{1} \varphi_{*}\left(T_{X} \otimes \Omega_{X}^{1}\right)\right) \geq h^{1}\left(C, T_{X} \otimes \Omega_{X}^{1} \mid C\right) .
$$

In fact, all cohomology classes in $H^{1}\left(C, T_{X} \otimes \Omega_{X}^{1} \mid C\right)$ extend to all infinitesimal neighborhoods, since $H^{2}\left(C,\left(N_{C}^{*}\right)^{\otimes k} \otimes T_{X} \otimes \Omega_{X}^{1} \mid C\right)=0$ for all $k$. Since

$$
h^{1}\left(C, T_{X} \otimes \Omega_{X}^{1} \mid C\right)=5
$$

in Case (2) and

$$
h^{1}\left(C, T_{X} \otimes \Omega_{X}^{1} \mid C\right)=7
$$


in Case (3), we need only to consider Case (1). In this case,

$$
h^{1}\left(C, T_{X} \otimes \Omega_{X}^{1} \mid C\right)=4 .
$$

Here we need to consider the second infinitesimal neighborhood $C_{2}$, defined by the ideal $\mathcal{I}_{C}^{2}$, and use the inequality

$$
h^{0}\left(Y, R^{1} \varphi_{*}\left(T_{X} \otimes \Omega_{X}^{1}\right)\right) \geq h^{1}\left(C_{2}, T_{X} \otimes \Omega_{X}^{1} \mid C_{2}\right) .
$$

The right hand side appears in the cohomology sequence

$$
\begin{gathered}
0 \rightarrow H^{0}\left(C, N_{C}^{*} \otimes T_{X} \otimes \Omega_{X}^{1} \mid C\right) \rightarrow H^{0}\left(C_{2}, T_{X} \otimes \Omega_{X}^{1} \mid C_{2}\right) \stackrel{\alpha}{\rightarrow} H^{0}\left(C, T_{X} \otimes \Omega_{X}^{1}\right) \rightarrow \\
\left.\rightarrow H^{1}\left(N_{C}^{*} \otimes T_{X} \otimes \Omega_{X}^{1}\right) \rightarrow H^{1}\left(C_{2}, T_{X} \otimes \Omega_{X}^{1}\right) \mid C_{2}\right) .
\end{gathered}
$$

By [Lau81, Thm.3.2], a sufficiently small neighborhood of $C \subset X$ is biholomorphic to a small neighborhood of the zero section of the normal bundle $N_{C}$, hence $\alpha$ is surjective. Since

$$
h^{1}\left(N_{C}^{*} \otimes T_{X} \otimes \Omega_{X}^{1}\right)=4,
$$

Claim (5.10.1) also holds in Case (1).

Since $\varphi$ is small, the sheaf $\varphi_{*}\left(T_{X} \otimes \Omega_{X}^{1}\right)$ is reflexive, hence

$$
\varphi_{*}\left(T_{X} \otimes \Omega_{X}^{1}\right)=\left(T_{Y} \otimes \Omega_{Y}^{[1]}\right)^{* *}=: \mathcal{F} .
$$

Since we assume $H^{1}\left(X, T_{X} \otimes \Omega_{X}^{1}\right)=0$, the Leray spectral sequence yields

$$
H^{1}(Y, \mathcal{F})=0
$$

further, the edge morphism

$$
\mu: E_{2}^{0,1} \rightarrow E_{2}^{2,0}
$$

is injective, hence by Claim (5.10.1),

$$
h^{2}(Y, \mathcal{F}) \geq h^{0}\left(Y, R^{1} \varphi_{*}\left(T_{X} \otimes \Omega_{X}^{1}\right)\right) \geq 5 .
$$

We now compute $H^{2}(Y, \mathcal{F})$ in a different way to obtain a contradiction. By Serre duality,

$$
H^{2}(Y, \mathcal{F}) \simeq \operatorname{Ext}^{1}\left(\mathcal{F}, \mathcal{O}_{Y}\right) .
$$

We will use the Grothendieck spectral sequence, with $E_{2}$-terms

$$
E_{2}^{p, q}=H^{p}\left(Y, \mathcal{E} x t^{q}\left(\mathcal{F}, \mathcal{O}_{Y}\right)\right)
$$

converging to $\operatorname{Ext}^{p+q}\left(\mathcal{F}, \mathcal{O}_{Y}\right)$. Notice that

$$
H^{p}\left(Y, \mathcal{E} x t^{0}\left(\mathcal{F}, \mathcal{O}_{Y}\right)\right)=H^{p}\left(Y, \mathcal{H o m}\left(\mathcal{F}, \mathcal{O}_{Y}\right)\right)=H^{p}(Y, \mathcal{F}),
$$

since $\mathcal{F} \simeq \mathcal{F}^{*}$. Thus, introducing the edge morphism $\delta: E_{2}^{0,1} \rightarrow E_{2}^{2,0}$, the spectral sequence together with the vanishing $E_{2}^{1,0}=0$ yields

$$
H^{2}(Y, \mathcal{F}) \simeq \operatorname{ker} \delta .
$$

Since $\operatorname{Ext}^{2}\left(\mathcal{F}, \mathcal{O}_{Y}\right)=H^{1}(Y, \mathcal{F})=0$, necessarily $E_{3}^{2,0}=0$, i.e., $E_{2}^{2,0}=\operatorname{im} \delta$, so the morphism $\delta$ is surjective. Since $E_{2}^{2,0}=H^{2}(Y, \mathcal{F}) \simeq \operatorname{ker} \delta$, we obtain

$$
2 h^{2}(Y, \mathcal{F})=\operatorname{dim} E_{2}^{0,1}=h^{0}\left(Y, \mathcal{E} x t^{1}\left(\mathcal{F}, \mathcal{O}_{Y}\right)\right) .
$$

The sheaf $\mathcal{E} x t^{1}\left(\mathcal{F}, \mathcal{O}_{Y}\right)$ being supported on $y_{0}$, we need to compute its length at $y_{0}$. Recalling that $y_{0}$ is a hypersurface singularity, $\Omega_{Y}^{1}=\Omega_{Y}^{[1]}$ by a theorem of Kunz [Kun86, Cor. 9.8], hence

$$
\mathcal{F}=\mathcal{H o m}\left(\Omega_{Y}^{1}, \Omega_{Y}^{1}\right)
$$


For our local computation, we may assume $Y$ itself to be a hypersurface in $\mathbb{C}^{4}$. We consider the cotangent sequence

$$
0 \rightarrow N_{Y / \mathbb{C}^{4}}^{*} \rightarrow \Omega_{\mathbb{C}^{4}}^{1} \mid Y \rightarrow \Omega_{Y}^{1} \rightarrow 0
$$

which after possibly shrinking $Y$ reads

$$
0 \rightarrow \mathcal{O}_{Y} \rightarrow \mathcal{O}_{Y}^{\oplus 4} \rightarrow \Omega_{Y}^{1} \rightarrow 0 .
$$

Tensoring by $T_{Y}$ gives

$$
0 \rightarrow T_{Y} \rightarrow T_{Y}^{\oplus 4} \rightarrow T_{Y} \otimes \Omega_{Y}^{1} \rightarrow 0 .
$$

The sheaf $T_{Y} \otimes \Omega_{Y}^{1}$ is clearly torsion free, as seen directly from the exact sequence (5.10.4), but possibly not reflexive. To see the difference, introduce the quotient $\mathcal{R}=\mathcal{F} /\left(T_{Y} \otimes \Omega_{Y}^{1}\right)$ which is supported on $y_{0}$. Dualizing the resulting exact sequence

$$
0 \rightarrow T_{Y} \otimes \Omega_{X}^{1} \rightarrow \mathcal{F} \rightarrow \mathcal{R} \rightarrow 0
$$

gives

$$
\mathcal{E} x t^{1}\left(\mathcal{R}, \mathcal{O}_{Y}\right) \rightarrow \mathcal{E} x t^{1}\left(\mathcal{F}, \mathcal{O}_{Y}\right) \rightarrow \mathcal{E} x t^{1}\left(T_{Y} \otimes \Omega_{Y}^{1}, \mathcal{O}_{Y}\right) .
$$

Since $\mathcal{R}$ is supported on $y_{0}$, we have $\mathcal{E} x t^{1}\left(\mathcal{R}, \mathcal{O}_{Y}\right)=0$, and thus it suffices to estimate $h^{0}\left(Y, \mathcal{E} x t^{1}\left(T_{Y} \otimes \Omega_{Y}^{1}, \mathcal{O}_{Y}\right)\right.$. Dualizing the exact sequence (5.10.4) yields the exact sequence

$$
\left(\Omega_{Y}^{1}\right)^{\oplus 4} \stackrel{\alpha}{\rightarrow} \Omega_{Y}^{1} \rightarrow \mathcal{E} x t^{1}\left(T_{Y} \otimes \Omega_{Y}^{1}, \mathcal{O}_{Y}\right) \rightarrow \mathcal{E} x t^{1}\left(\Omega_{Y}^{1}, \mathcal{O}_{Y}\right)^{\oplus 4} .
$$

Since $\alpha$ is simply the map

$$
\operatorname{id} \otimes(d f)^{*}: \Omega_{Y}^{1} \otimes \mathcal{O}_{Y}^{\oplus 4} \rightarrow \Omega_{Y}^{1} \otimes \mathcal{O}_{Y}=\Omega_{Y}^{1},
$$

where $f$ is the equation for $Y \subset \mathbb{C}^{4}$, it follows

$$
\operatorname{coker} \alpha=\mathcal{O}_{y_{0}}^{\oplus 4} .
$$

Dualizing the cotangent sequence and using the same argument gives also

$$
\mathcal{E} x t^{1}\left(\Omega_{Y}^{1}, \mathcal{O}_{Y}\right)=\mathcal{O}_{y_{0}} .
$$

Hence in total,

$$
h^{0}\left(Y, \mathcal{E} x t^{1}\left(T_{Y} \otimes \Omega_{Y}^{1}, \mathcal{O}_{Y}\right)\right) \leq 8,
$$

and consequently by Equation (5.10.3),

$$
h^{2}(Y, \mathcal{F})=\frac{1}{2} h^{0}\left(Y, \mathcal{E} x t^{1}\left(\mathcal{F}, \mathcal{O}_{Y}\right)\right) \leq 4 .
$$

This contradicts Inequality (5.10.2), completing the proof of Proposition 5.10 ,

5.11. Corollary. Let $X$ be a Calabi-Yau threefold and $C \subset X$ be a smooth rational curve with normal bundle $N_{C}$. Assume either that $N_{C}=\mathcal{O}_{C}(-1) \oplus \mathcal{O}_{C}(-1)$ or that $N_{C}=\mathcal{O}_{C} \oplus \mathcal{O}_{C}(-2)$ and that $C$ is an isolated curve in the sense of [Rei83], i.e., $C$ does not move in $X$. Then $H^{1}\left(X, T_{X} \otimes \Omega_{X}^{1}\right) \neq 0$.

Proof. By Proposition 5.10 it suffices to prove that $C$ is contractible. In the first case this is Grauert's criterion Gra62; in the second case we apply a theorem of Reid [Rei83, Cor. 5.6].

We now apply Corollary 5.11 to compute $H^{1}\left(X, T_{X} \otimes \Omega_{X}^{1}\right)$. 
5.12. Theorem. Let $\varphi: X \rightarrow Y$ be a primitive contraction of the Calabi-Yau threefold with exceptional divisor $E$. Assume that $\operatorname{dim} \varphi(E)=0$ and that one of the following conditions holds.

(1) $E$ is smooth;

(2) $K_{E}^{2} \geq 6$;

(3) $E$ is normal, rational and contains a smooth contractible rational curve, e.g., E carries a birational contraction of an extremal ray;

(4) $E$ is normal and irrational;

(5) $E$ is non-normal.

Then

$$
H^{1}\left(X, \Omega_{X}^{1} \otimes T_{X}\right) \neq 0 .
$$

Proof. (1) If $E$ is smooth and if $K_{E}^{2} \geq 6$, the claim follows from Theorem 5.8, combined with Remark [5.9, since $E$ is a del Pezzo surface. If $E$ is smooth with $K_{E}^{2} \leq 7$, then we may choose a $(-1)$-curve $C \subset E$. Then, using the normal bundle sequence for $C \subset E \subset X$, it is immediate that $C$ has normal bundle $N_{C / X}=\mathcal{O}_{C}(-1) \oplus \mathcal{O}_{C}(-1)$. Hence Corollary 5.11 applies.

(2) By a theorem of Gross, Gro97a, proof of 5.8] and Wilson, Wil97, p.620-624], there exists an open neighborhood $U=U_{0} \subset X$ of $E_{0}:=E$ and a deformation

$$
\pi: \mathcal{U} \rightarrow \Delta
$$

over the unit disc and a divisor $\mathcal{E} \subset \mathcal{U}$ such that $X_{0}=\pi^{-1}(0)$, such that $\mathcal{E} \cap X_{0}=E_{0}$ and such that - after possibly shrinkling $\Delta$ - the divisor $E_{t}=\mathcal{E} \cap X_{t}$ is smooth. Since the normal bundle $N_{E / X}$ is negative, so does $N_{E_{t} / U_{t}}$, hence $E_{t}$ is contractible. Thus, we obtain a family $\phi_{t}: U_{t} \rightarrow V_{t}$ contracting fiberwise the divisor $E_{t}$. Notice that

$$
K_{U_{t}} \simeq \mathcal{O}_{U_{t}}
$$

for all $t$. In fact, we may choose $U_{t}$ such that $E_{t}$ is a deformation retract of $U_{t}$. Hence the restriction

$$
H^{2}\left(U_{t}, \mathbb{Z}\right) \rightarrow H^{2}\left(E_{t}, \mathbb{Z}\right)
$$

is an isomorphism. Since $H^{q}\left(E_{t}, \mathcal{O}_{E_{t}}\right)=0$, the restriction

$$
\operatorname{Pic}\left(U_{t}\right) \rightarrow \operatorname{Pic}\left(E_{t}\right)
$$

is an isomorphism, too. Since $K_{X} \mid E \simeq \mathcal{O}_{E}$, it follows that $K_{U_{t}} \mid E_{t} \equiv 0$, hence $K_{U_{t}} \mid E_{t} \simeq \mathcal{O}_{E_{t}}$. Hence Equation (5.12.1) follows.

We assume now that $K_{E}^{2} \geq 6$. Using the inclusion

$$
N_{E_{t} / U_{t}}^{*} \subset \Omega_{U_{t}}^{1},
$$

and observing $N_{E_{t} / U_{t}}^{*} \simeq \mathcal{O}_{E_{t}}\left(-K_{E_{t}}\right)$, we have an inclusion

$$
\begin{gathered}
H^{0}\left(E_{t}, T_{E_{t}}\right)=H^{0}\left(E_{t}, T_{E_{t}} \otimes N_{E_{t} / U_{t}}^{*} \otimes \mathcal{O}_{E_{t}}\left(K_{E_{t}}\right)\right) \rightarrow \\
\rightarrow H^{0}\left(E_{t}, T_{U_{t}} \otimes \Omega_{U_{t}}^{1} \mid E_{t} \otimes \mathcal{O}_{E_{t}}\left(K_{E_{t}}\right)\right) .
\end{gathered}
$$

Since $K_{E_{t}}^{2}=K_{E}^{2} \geq 6$, we conclude Observe that, using the conormal sheaf sequence and the triviality of $K_{U_{t}}$ that

$$
H^{0}\left(E_{t}, T_{U_{t}} \otimes \Omega_{U_{t}}^{1} \mid E_{t} \otimes \mathcal{O}_{E_{t}}\left(K_{E_{t}}\right)\right)=\neq 0 .
$$


By semi-continuity,

$$
H^{0}\left(E, T_{U} \otimes \Omega_{U}^{1} \mid E \otimes \mathcal{O}_{E}\left(K_{E}\right)\right) \neq 0 .
$$

By Serre duality,

$$
H^{2}\left(E, T_{X} \otimes \Omega_{X}^{1} \mid E\right) \neq 0,
$$

and we conclude by Lemma 5.7 .

(3) Suppose now that $K_{E}^{2} \leq 5$ and $E$ is normal and rational (but singular). Then by [HW81, $E$ is either a rational surface with only ADE singularities or an elliptic cone; hence in our case, the first alternative holds. Let $C \subset E$ be a smooth contractible rational curve; $C$ is a $\mathbb{Q}$-divisor in $E$, but possibly not Cartier. The conormal sheaf $N_{C / E}^{*}$ is of the form

$$
N_{C / E}^{*}=\mathcal{O}_{C}(a) \oplus \mathcal{T}
$$

with $a \geq 0$ and $\mathcal{T}$ a torsion sheaf, supported on $C \cap \operatorname{Sing}(E)$. Consider the conormal sheaf sequence

$$
0 \rightarrow N_{E / X}^{*} \mid C \rightarrow N_{C / X}^{*} \rightarrow N_{C / E}^{*} \rightarrow 0 .
$$

Since $N_{E / X}^{*}\left|C=-K_{E}\right| C$ is ample, either $a=1$ and $N_{C / X}^{*}=\mathcal{O}_{C}(1) \oplus \mathcal{O}_{C}(1)$, or $a=0, \mathcal{T}$ is supported on one point with one-dimensional stalk and $N_{C / X}^{*}=$ $\mathcal{O}_{C}(2) \oplus \mathcal{O}_{C}$. In both cases we conclude by Corollary 5.11

(4) If $E$ is normal with a non-rational singularity, then, as already mentioned, $E$ is an elliptic cone. In this case, $H^{0}\left(E, T_{E}\right) \neq 0$, and we conclude by Theorem 5.8 ,

(5) Suppose finally that $E$ is non-normal. In case $y_{0}$ is not a hypersurface singularity, $K_{E}^{2}=7$ by Gro97a, Thm. 5.2]. This case is settled by (2). Thus we may assume that $y_{0}$ is a hypersurface singularity. Then

$$
1 \leq K_{E}^{2} \leq 3
$$

by Gro97a, p.201], see also Wil97, p.620]. Non-normal del Pezzo surfaces being classified by Reid Rei94, we show case by case that $H^{0}\left(E, T_{E}\right) \neq 0$. Then we conclude by Theorem 5.8 Actually, we have more informations on $E$, Gro97a, p.201] and Wil97, p.620]. In fact, if $K_{E}^{2}=1$, then $E$ is a hypersurface in the weighted projective space $\mathbb{P}(3,1,1,1)$ with explicit equation, in case $K_{E}^{2}$ the surface $E$ is a hypersurface in $\mathbb{P}(2,1,1,1)$ and if $K_{E}^{2}=3$, then $E$ is a cubic. The normalization $\widetilde{E}$ in the last two cases are Hirzebruch surfaces, while the normalization in the first case is $\mathbb{P}_{2}$.

We give two examples. First assume that $K_{E}^{2}=3$; so $E$ is a cubic surface in $\mathbb{P}_{3}$. The nonnormal locus $N$ (with the structure given by the conductor ideal) is a line in $E$. Then the tangent sheaf sequence reads

$$
0 \rightarrow T_{E} \rightarrow T_{\mathbb{P}_{3}} \mid E \rightarrow \mathcal{I}_{N} \otimes N_{E / \mathbb{P}_{3}} \rightarrow 0
$$

(actually it suffices that the image of $T_{E} \rightarrow T_{\mathbb{P}_{3}} \mid E$ is contained in $\mathcal{I}_{N} \otimes N_{E / \mathbb{P}_{3}}$ ). Now $h^{0}\left(E, T_{\mathbb{P}_{3}} \mid E\right)=15$ and $h^{0}\left(E, N_{E / \mathbb{P}_{3}}\right)=h^{0}\left(E, \mathcal{O}_{E}(3)\right)=19$. Since the image of the restriction map $H^{0}\left(E, \mathcal{O}_{E}(3)\right) \rightarrow H^{0}\left(N, \mathcal{O}_{N}(3)\right)$ is surjective, it has dimension 5 , hence $h^{0}\left(E, \mathcal{I}_{N} \otimes N_{E / \mathbb{P}_{3}}\right)=14$, hence $h^{0}\left(E, T_{E}\right) \neq 0$.

Second, consider the case $K_{E}^{2}=1$, hence $\widetilde{E} \simeq \mathbb{P}_{2}$. Let $\eta: \widetilde{E} \rightarrow E$ be the normalization map; the non-normal locus $N$ of $E$ is a line. We consider the subcase when the preimage $\widetilde{N}$ is a smooth conic; it might also be a line pair or a double line. The degree of $\eta \mid \widetilde{N} \rightarrow N$ is two. Then, $H^{0}\left(\widetilde{E}, T_{\widetilde{E}}\right)=H^{0}\left(\widetilde{N}, T_{\widetilde{E}} \mid \widetilde{N}\right)$ and the 
latter contains $H^{0}\left(\widetilde{N}, T_{\widetilde{N}}\right)$. Thus there are three vectors fields on $\widetilde{E}$, tangent to $\widetilde{N}$ and one of them is invariant under the double cover $\widetilde{N} \rightarrow N$ (by considering $\left.T_{\widetilde{N}} \rightarrow \eta^{*}\left(T_{N}\right)\right)$.

5.13. Remark. The remaning open case in Theorem $[5.12$ is the following: $E$ is normal, but singular, rational with only ADE-singularities containing no contractible smooth rational curve and $K_{E}^{2} \leq 5$. Then necessarily $\rho(E) \leq 2$, otherwise $E$ carries a birational contraction of an extremal ray. The way to treat this open case would be to show that there is a global deformation $X_{t}$ of $X$ (not only a local deformation of a neighborhood of $E$ ), such that $E$ deforms to a smooth del Pezzo surface $E_{t}$ and then to conclude again by semicontinuity.

We now turn to the case that $E$ is contracted to a curve $C$. We already know that $T_{X}$ has a first order deformation if $E$ is smooth with $H^{0}\left(E, T_{E}\right) \neq 0$, e.g., if $C \simeq \mathbb{P}_{1}$. The case that $C$ is an elliptic curve is easy as well, provided $c_{3}(X) \neq 4$ :

5.14. Proposition. Let $X$ be a Calabi-Yau threefold, $\varphi: X \rightarrow Y$ be a primitive contraction with smooth exceptional divisor $E$ such that $C=\varphi(E)$ is a curve. If $g(C)=1$ and if $c_{3}(X) \neq 4$, then $H^{1}\left(X, \Omega_{X}^{1} \otimes T_{X}\right) \neq 0$.

Proof. By Lemma 5.7 and Remark 5.5 it suffices to show

$$
H^{2}\left(E, \Omega_{X}^{1} \otimes T_{X} \mid E\right) \neq 0
$$

By Serre duality, this is equivalent to

$$
H^{0}\left(E, \Omega_{X}^{1} \otimes T_{X} \mid E \otimes K_{E}\right) \neq 0 .
$$

Using the tangent bundle sequence, it suffices to show that

$$
H^{0}\left(E, \Omega_{X}^{1} \otimes T_{E} \otimes K_{E}\right)=H^{0}\left(E, \Omega_{X}^{1} \otimes \Omega_{E}^{1}\right) \neq 0 .
$$

By the cotangent sequence, we obtain an exact sequence

$$
\begin{gathered}
H^{0}\left(E, T_{E}\right) \rightarrow H^{0}\left(E, \Omega_{X}^{1} \otimes \Omega_{E}^{1}\right) \rightarrow \\
\rightarrow H^{0}\left(E, \Omega_{E}^{1} \otimes \Omega_{E}^{1}\right) \stackrel{\delta}{\rightarrow} H^{1}\left(E, N_{E}^{*} \otimes \Omega_{E}^{1}\right) \simeq H^{1}\left(E, T_{E}\right) .
\end{gathered}
$$

Since we may assume $H^{0}\left(E, T_{E}\right)=0$ by Theorem 5.8 and since clearly $H^{2}\left(E, T_{E}\right)=$ 0 , Riemann-Roch shows, using $g(C)=1$, that

$$
H^{1}\left(E, T_{E}\right)=0 \text {. }
$$

Since

$$
H^{0}\left(E, \Omega_{E}^{1} \otimes \Omega_{E}^{1}\right) \neq 0
$$

our claim follows.

5.15. Remark. If $g(C) \geq 2$, these simple arguments do no longer work (provided $\Omega_{Y}^{[1]}$ is $H$-stable). The difficulty is that $\operatorname{dim} H^{1}\left(E, T_{E}\right)=6(g-1)$, assuming $H^{0}\left(E, T_{E}\right)=0$, whereas

$$
\operatorname{dim} H^{0}\left(E, \Omega_{E}^{1} \otimes \Omega_{E}^{1}\right)=3(g-1) .
$$

One would need to show that the connecting map

$$
\delta: H^{0}\left(E, \Omega_{E}^{1} \otimes \Omega_{E}^{1}\right) \rightarrow H^{1}\left(E, N_{E}^{*} \otimes \Omega_{E}^{1}\right) \simeq H^{1}\left(E, T_{E}\right)
$$


is not injective. Actually, this statement can still be sharpened. In fact, assuming as always that $H^{0}\left(E, T_{E}\right)=0$, then the sequence

$$
0 \rightarrow T_{E / C} \rightarrow T_{E} \rightarrow \varphi^{*}\left(T_{C}\right) \rightarrow 0
$$

splits. Thus it suffices to show that

$$
H^{0}\left(E, \Omega_{X}^{1} \mid E \otimes \varphi^{*}\left(T_{C}\right)\right) \neq 0 .
$$

This comes down to show that the canonical morphism

$$
\epsilon: H^{0}\left(E, \Omega_{E}^{1} \otimes \varphi^{*}\left(T_{C}\right)\right) \rightarrow H^{1}\left(E, N_{E}^{*} \otimes \Omega_{E}^{1}\right)
$$

is not injective. Notice also that both vector spaces have the same dimension $3(g(C)-1)$.

We treat next the case $g(C) \geq 2$ and the case that $E$ is singular by a more sophisticated method.

5.16. Theorem. Let $X$ be a Calabi-Yau threefold, $\varphi: X \rightarrow Y$ a primitive divisorial contraction contracting the exceptional divisor $E$ to a curve $C$. Then $H^{1}\left(X, T_{X} \otimes \Omega_{X}^{1}\right) \neq 0$ unless (possibly) $E$ is one of the following surfaces

(1) $E$ is a normal singular surface and all singular fibers are double lines;

(2) $E$ is a non-normal surface, $C \simeq \mathbb{P}_{1}$, but the normalization of $E$ is irrational.

Proof. Recall that $p:=\varphi_{\mid E}: E \rightarrow C$ is a conic bundle over the smooth curve $C$. We will also use the finer classification of $E$, due to Wilson Wil92, Wil93, Wil97. In fact, consider a singular fiber $E_{c}$ of $p$ which is a line pair $E_{c}=C_{1} \cup C_{2}$ (with $C_{1} \neq C_{2}$ ). Then there are three possibilities:

- $E$ is smooth along $C_{1} \cup C_{2}$;

- $E$ is normal along along $C_{1} \cup C_{2}$ and has an $A_{n}$-singularity at the intersection point $C_{1} \cap C_{2}$ and is smooth elsewhere;

- $E$ is non-normal, the normalisation is a $\mathbb{P}_{1}$-bundle over a smooth curve $\tilde{C}$ which is a double cover over $C$ and unramified over $c$. The singular locus of $E$ meets $C_{1}$ exactly in the intersection point $C_{1} \cap C_{2}$.

(A) Assume first that the general fiber of $p$ is irreducible, but $p$ has a reducible fiber $E_{c}$, and fix an irreducible component $B \simeq \mathbb{P}_{1}$. Then the conormal sheaf sequence

$$
0 \rightarrow N_{E / X}^{*} \mid B \rightarrow N_{B / X}^{*} \rightarrow N_{B / E}^{*} \rightarrow 0
$$

implies - together with the equation $E \cdot B=-1$ - that $N_{B / X}^{*}$ is either $\mathcal{O}_{B}(1) \oplus \mathcal{O}_{B}(1)$ or $\mathcal{O}_{B}(2) \oplus \mathcal{O}_{B}$. In both cases $B$ is contractible; in the first case by Grauert's criterion Gra62, in the second case we apply again Rei83, Cor. 5.6], using our assumption that the general fiber of $p$ is irreducible, hence $B$ does not move. We also use the sequence

$$
0 \rightarrow N_{E_{c} / E}^{*} \mid B \simeq \mathcal{O}_{B} \rightarrow N_{B / E}^{*} \rightarrow N_{B / E_{c}}^{*} \rightarrow 0
$$

to conclude that either $N_{B / E}^{*} \simeq \mathcal{O}_{B}(1)$ or that $N_{B / E}^{*} \simeq \mathcal{O}_{B} \oplus \mathcal{O}_{x}$, where $\mathcal{O}_{x}$ is a sheaf supported on the singularity of $E_{c}$ with one-dimensional stalk at $x$. Once we know that $B$ is contractible, we conclude by Proposition 5.10 .

(B) Next we consider the case $g(C) \geq 1$. By [Gro97b, 1.2,1.3], Wil97, p.631 ff] there exists a flat family

$$
\pi: \mathcal{X} \rightarrow \Delta
$$

of Calabi-Yau threefolds $X_{t}$ over the unit disc with the following properties 
(1) $X_{0}=\pi^{-1}(0) \simeq X$;

(2) there is a relative crepant contraction $\Phi: \mathcal{X} \rightarrow \mathcal{Y}$ over $\Delta$ with $\Phi \mid X_{0}=\varphi$;

(3) $\varphi_{t}=\Phi \mid X_{t}: X_{t} \rightarrow Y_{t}$ is small;

(4) $\varphi_{t}$ contracts the deformations of the finitely many fibers of $E \rightarrow C$ which deform to $X_{t}$.

By Theorem 5.18, $H^{1}\left(X_{t}, T_{X_{t}} \otimes \Omega_{X_{t}}^{1}\right) \neq 0$, hence $H^{1}\left(X, T_{X} \otimes \Omega_{X}^{1}\right) \neq 0$ by semicontinuity.

(C) We next consider the case that $C \simeq \mathbb{P}_{1}$ and that all fibers of $p: E \rightarrow C$ are irreducible. If $E$ is smooth, $p$ is a $\mathbb{P}_{1}$-bundle, and we are done by Lemma [5.4] Theorem 5.8 in connection with Remark 5.9. If $E$ is singular, then $E$ is normal and by (A), we may assume that the only singular fibers are double lines. This case is ruled out by assumption.

(D) Assume finally that the general fiber of $p$ is reducible. Then there is a double cover $\tilde{C} \rightarrow C$ such that the fiber product $\tilde{E} \rightarrow \tilde{C}$ is a $\mathbb{P}_{1}$-bundle. If $g(C)>0$, then by [Wil97, p.635] and [Gro97b, p.294], the general deformation $X_{t}$ of $X$ carries a small contraction. Hence

$$
H^{1}\left(X_{t}, T_{X_{t}} \otimes \Omega_{X_{t}}^{1}\right) \neq 0
$$

by Theorem [5.18, and we conclude by semicontinuity.

If $g(\tilde{C})=0$, then by Wil97, p.635], the non-normal locus of $E$ is a $(-1,-1)$-curve and we conclude by Proposition 5.10 Finally, the case $g(\tilde{C})>0$ and $g(C)=0$ is ruled out by assumption.

5.17. Remark. The exceptional case (b) might be ruled out as follows. Consider again a general deformation $X_{t}$ of $X$. Then $E$ deforms to a rational surface which is a conic bundle over $\mathbb{P}_{1}$; see Wi197, p.635]. One might expect that not all singular fibers are double lines, hence $H^{1}\left(X_{t}, T_{X_{t}} \otimes \Omega_{X_{t}}^{1}\right) \neq 0$ and we conclude again by semicontinuity. Thus the difficulty is that in all deformation $E_{t} \subset X_{t}$, we land in the exceptional case (1).

We finally consider small contractions $\varphi: X \rightarrow Y$.

5.18. Theorem. Let $X$ be a Calabi-Yau threefold and $\varphi: X \rightarrow Y$ be a small contraction. Then $H^{1}\left(X, T_{X} \otimes \Omega_{X}^{1}\right) \neq 0$.

Proof. By [Lau81, Pin83, Mor85, Thm.5.5], see also [Fri86, any fiber $F$ of $\varphi$ has the form $F=\cup C_{j}$ with smooth rational curves $C_{j}$. All $C_{j}$ have normal bundle

$$
N_{C / X}=\mathcal{O}_{C}(a) \oplus \mathcal{O}_{C}(b)
$$

with $(a, b)=(-1,-1),(0,-2),(1,-3)$. Moreover, there is at most one curve with $(a, b)=(1,-3)$. Thus we conclude by Corollary 5.11 applied to some component $C_{j}$ whose normal bundle is not of type $(1,-3)$ - unless $F=C_{1}$ is irreducible with normal bundle of type $(1,-3)$. Then we apply Proposition 5.10 .

Any small contraction $\varphi: X \rightarrow Y$ gives rise to a flop $h: X \rightarrow X^{+}$with a (smooth) Calabi-Yau threefold $X^{+}$, Kol89, 2.4]. We finally relate the deformations of $T_{X}$ to those of $T_{X^{+}}$. 
5.19. Proposition. Let $h: X \rightarrow X^{+}$be a flop of the Calabi-Yau threefold $X$, induced by the small contraction $\varphi: X \rightarrow Y$. Then

$$
H^{1}\left(X, T_{X} \otimes \Omega_{X}^{1}\right) \simeq H^{1}\left(X, T_{X^{+}} \otimes \Omega_{X^{+}}^{1}\right) .
$$

Moreover, every positive-dimensional deformation of $T_{X}$ over (a germ of) an irreducible reduced complex space $S$ induces canonically a positive-dimensional deformation of $T_{X}+$ over $S$.

Proof. In order to show the first claim, it suffices to show that

$$
\left.H^{2} X, T_{X} \otimes \Omega_{X}^{1}\right) \simeq H^{2}\left(X, T_{X^{+}} \otimes \Omega_{X^{+}}^{1}\right) .
$$

The flop being induced by a small contraction $\varphi: X \rightarrow Y$, we let $\varphi^{+}: X^{+} \rightarrow Y$ denote the associated flopped small morphism. The Leray spectral sequence gives

$$
H^{2}\left(X, T_{X} \otimes \Omega_{X}^{1}\right) \simeq H^{2}\left(Y, \varphi_{*}^{+}\left(T_{X} \otimes \Omega_{X}^{1}\right)\right)
$$

and

$$
H^{2}\left(X^{+}, T_{X^{+}} \otimes \Omega_{X^{+}}^{1}\right) \simeq H^{2}\left(Y, \varphi_{*}^{+}\left(T_{X^{+}} \otimes \Omega_{X^{+}}^{1}\right)\right) .
$$

The sheaves $\varphi_{*}\left(T_{X} \otimes \Omega_{X}^{1}\right)$ and $\varphi_{*}^{+}\left(T_{X^{+}} \otimes \Omega_{X^{+}}^{1}\right)$ are reflexive and isomorphic outside a finite set. Hence they are isomorphic on all of $Y$, and the claim follows.

As to the second claim, let $\mathcal{E}$ be a flat deformation of $T_{X}$ over $X \times S$. The locally free sheaf $\mathcal{E}$ induces a coherent sheaf $\mathcal{E}^{+}$over $X^{+} \times S$, such that $\left(\mathcal{E}^{+}\right)_{\mid X^{+} \times\{0\}} \simeq T_{X^{+}}$. In particular, $\left(\mathcal{E}^{+}\right)_{\mid X^{+} \times\{0\}}$ is locally free and so does $\left(\mathcal{E}^{+}\right)_{\mid X^{+} \times\{s\}}$ for small $s$. Hence $\mathcal{E}^{+}$is flat over $S$.

5.20. Corollary. Let $X$ and $X^{\prime}$ be birationally equivalent Calabi-Yau threefolds. Then

$$
H^{1}\left(X, T_{X} \otimes \Omega_{X}^{1}\right) \simeq H^{1}\left(X, T_{X^{\prime}} \otimes \Omega_{X^{\prime}}^{1}\right) .
$$

Moreover, every positive-dimensional deformation of $T_{X}$ induces canonically a positive-dimensional deformation of $T_{X^{\prime}}$.

Proof. It suffices to remark that any birational map between (smooth) Calabi-Yau threefolds is a sequence of flops, Kol89.

\section{Higher Dimensions}

We finish the paper with some results in higher dimensions: hypersurfaces in projective space, and products, the latter being important for the correct set-up of Question 1.6

6.1. Theorem. Let $X \subset \mathbb{P}_{n+1}, n \geq 2$, be a smooth hypersurface of degree $d \geq 2$. Then $T_{X}$ has a genuine first order deformation.

Proof. Since $H^{1}\left(X, \mathcal{O}_{X}\right)=0$, it suffices to show that $H^{1}\left(X, T_{X} \otimes \Omega_{X}^{1}\right) \neq 0$. We use the cohomology sequence

$$
\begin{aligned}
0 \rightarrow H^{0}\left(X, T_{X} \otimes \Omega_{X}^{1}\right) & \rightarrow H^{0}\left(X,\left.T_{\mathbb{P}_{n+1}}\right|_{X} \otimes \Omega_{X}^{1}\right) \rightarrow H^{0}\left(X, \Omega_{X}^{1}(d)\right) \rightarrow \\
& \rightarrow H^{1}\left(X, T_{X} \otimes \Omega_{X}^{1}\right) .
\end{aligned}
$$

The Euler sequence in combination with the vanishing $H^{1}\left(X, \Omega_{X}^{1}(1)\right)=0$ (use the cotangent sequence for $X \subset \mathbb{P}_{n+1}$ ) yields

$$
h^{0}\left(X,\left.T_{\mathbb{P}_{n+1}}\right|_{X} \otimes \Omega_{X}^{1}\right)=1 .
$$


Thus

$$
H^{0}\left(X, \Omega_{X}^{1}(d)\right) \subset H^{1}\left(X, T_{X} \otimes \Omega_{X}^{1}\right)
$$

and it suffices to observe that $H^{0}\left(X, \Omega_{X}^{1}(d)\right) \neq 0$, which is clear since $\Omega_{\mathbb{P}_{n+1}}^{1}(2)$ is generated by global sections.

Concerning products, we first consider the case of two factors.

6.2. Proposition. Let $X=X_{1} \times X_{2}$ be a projective manifold with $\operatorname{dim} X_{j} \geq 1$. Then

$$
H^{1}\left(X, \mathcal{E} n d_{0}\left(T_{X}\right)\right)=0
$$

if and only if the following conditions hold for $j=1,2$.

(1) $H^{0}\left(X_{j}, T_{X_{j}}\right)=0$;

(2) $q\left(X_{j}\right)=0$;

(3) $H^{1}\left(X_{j}, T_{X_{j}} \otimes \Omega_{X_{j}}^{1}\right)=H^{1}\left(X_{j}, \mathcal{E} n d_{0}\left(T_{X_{j}}\right)\right)=0$.

In particular, $\operatorname{dim} X_{j} \geq 2$ for $j=1,2$.

Proof. Let $p_{j}: X \rightarrow X_{j}$ denote the projections. Then

$$
\begin{gathered}
T_{X} \otimes \Omega_{X}^{1} \simeq\left(p_{1}^{*}\left(T_{X_{1}} \otimes \Omega_{X_{1}}^{1}\right)\right) \oplus\left(p_{1}^{*} T_{X_{1}} \otimes p_{2}^{*} \Omega_{X_{2}}^{1}\right) \oplus \\
\oplus\left(p_{2}^{*} T_{X_{2}} \otimes p_{1}^{*} \Omega_{X_{1}}^{1}\right) \oplus\left(p_{2}^{*}\left(T_{X_{2}} \otimes \Omega_{X_{2}}^{1}\right)\right) .
\end{gathered}
$$

Using the Künneth formula, a direct computation shows that the conditions (1),(2) and (3) are equivalent to $h^{1}\left(X, T_{X} \otimes \Omega_{X}^{1}\right)=q(X)$, i.e., $h^{1}\left(X, \mathcal{E} n d_{0}\left(T_{X}\right)\right)=0$. In particular, $\operatorname{dim} X_{j}=1$ is impossible.

Inductively, we obtain

6.3. Corollary. Let $X=\Pi_{j=1}^{m} X_{j}$ be a projective manifold. Then

$$
H^{1}\left(X, \mathcal{E} n d_{0}\left(T_{X}\right)\right)=0
$$

if and only if the following holds for all $j$.

(1) $H^{0}\left(X_{j}, T_{X_{j}}\right)=0$;

(2) $q\left(X_{j}\right)=0$;

(3) $H^{1}\left(X_{j}, \mathcal{E} n d_{0}\left(T_{X_{j}}\right)\right)=0$.

\section{REFERENCES}

[Bla96] Raimund Blache, Chern classes and Hirzebruch-Riemann-Roch theorem for coherent sheaves on complex-projective orbifolds with isolated singularities, Math. Z. 222 (1996), no. 1, 7-57. MR 1388002

[CWY03] Wing-Sum Cheung, Bun Wong, and Stephen S.-T. Yau, Some remarks on the local moduli of tangent bundles over complex surfaces, Amer. J. Math. 125 (2003), no. 5, 1029-1035. MR 2004427

[DGKM89] Jacques Distler, Brian R. Greene, Kelley Kirklin, and Paul Miron, Calculating endomorphism valued cohomology: singlet spectrum in superstring models, Comm. Math. Phys. 122 (1989), no. 1, 117-124. MR 994498

[EH90] Michael G. Eastwood and Tristan Hübsch, Endomorphism valued cohomology and gauge-neutral matter, Comm. Math. Phys. 132 (1990), no. 2, 383-413. MR 1069828

[Ele82] G. Elencwajg, The Brauer groups in complex geometry, Brauer groups in ring theory and algebraic geometry (Wilrijk, 1981), Lecture Notes in Math., vol. 917, Springer, Berlin-New York, 1982, pp. 222-230. MR 657432

[Fri86] Robert Friedman, Simultaneous resolution of threefold double points, Math. Ann. 274 (1986), no. 4, 671-689. MR 848512 
[GKKP11] Daniel Greb, Stefan Kebekus, Sándor J. Kovács, and Thomas Peternell, Differential forms on log canonical spaces, Publ. Math. Inst. Hautes Études Sci. (2011), no. 114, 87-169. MR 2854859

[GKP16] Daniel Greb, Stefan Kebekus, and Thomas Peternell, Singular spaces with trivial canonical class, Minimal models and extremal rays (Kyoto, 2011), Adv. Stud. Pure Math., vol. 70, Math. Soc. Japan, [Tokyo], 2016, pp. 67-113. MR 3617779

[Gra62] Hans Grauert, Über Modifikationen und exzeptionelle analytische Mengen, Math. Ann. 146 (1962), 331-368. MR 0137127

[Gro97a] Mark Gross, Deforming Calabi-Yau threefolds, Math. Ann. 308 (1997), no. 2, 187220. MR 1464900

[Gro97b] _ Primitive Calabi-Yau threefolds, J. Differential Geom. 45 (1997), no. 2, 288318. MR 1449974

[Gue16] Henri Guenancia, Semistability of the tangent sheaf of singular varieties, Algebr. Geom. 3 (2016), no. 5, 508-542. MR 3568336

[Har77] Robin Hartshorne, Algebraic geometry, Springer-Verlag, New York, 1977, Graduate Texts in Mathematics, No. 52. MR MR0463157 (57 \#3116)

[HP19] Andreas Höring and Thomas Peternell, Algebraic integrability of foliations with numerically trivial canonical bundle, Invent. Math. 216 (2019), no. 2, 395-419. MR 3953506

[Huy95] D. Huybrechts, The tangent bundle of a Calabi-Yau manifold-deformations and restriction to rational curves, Comm. Math. Phys. 171 (1995), no. 1, 139-158. MR 1341697

[HW81] Fumio Hidaka and Keiichi Watanabe, Normal Gorenstein surfaces with ample anticanonical divisor, Tokyo J. Math. 4 (1981), no. 2, 319-330. MR 646042

[Ino74] Masahisa Inoue, On surfaces of Class $\mathrm{VII}_{0}$, Invent. Math. 24 (1974), 269-310. MR 342734

[IP99] Vasily A. Iskovskih and Yuri G. Prokhorov, Fano varieties, Algebraic geometry, V, Encyclopaedia Math. Sci., vol. 47, Springer, Berlin, 1999, pp. 1-247. MR 1668579

[Kol89] János Kollár, Flops, Nagoya Math. J. 113 (1989), 15-36. MR 986434

[Kol96] János Kollár, Rational curves on algebraic varieties, Ergebnisse der Mathematik und ihrer Grenzgebiete. 3. Folge. A Series of Modern Surveys in Mathematics [Results in Mathematics and Related Areas. 3rd Series. A Series of Modern Surveys in Mathematics], vol. 32, Springer-Verlag, Berlin, 1996. MR 1440180

[Kun86] Ernst Kunz, Kähler differentials, Advanced Lectures in Mathematics, Friedr. Vieweg \& Sohn, Braunschweig, 1986. MR 864975

[Lau81] Henry B. Laufer, On $\mathbf{C} P^{1}$ as an exceptional set, Recent developments in several complex variables (Proc. Conf., Princeton Univ., Princeton, N. J., 1979), Ann. of Math. Stud., vol. 100, Princeton Univ. Press, Princeton, N.J., 1981, pp. 261-275. MR 627762

[LOP18] Vladimir Lazić, Keiji Oguiso, and Thomas Peternell, The Morrison-Kawamata cone conjecture and abundance on Ricci flat manifolds, Uniformization, Riemann-Hilbert correspondence, Calabi-Yau manifolds \& Picard-Fuchs equations, Adv. Lect. Math. (ALM), vol. 42, Int. Press, Somerville, MA, 2018, pp. 157-185. MR 3822906

[Mor85] David R. Morrison, The birational geometry of surfaces with rational double points, Math. Ann. 271 (1985), no. 3, 415-438. MR 787190

[Pin83] Henry C. Pinkham, Factorization of birational maps in dimension 3, Singularities, Part 2 (Arcata, Calif., 1981), Proc. Sympos. Pure Math., vol. 40, Amer. Math. Soc., Providence, RI, 1983, pp. 343-371. MR 713260

[Pro05] Yu. G. Prokhorov, The degree of Fano threefolds with canonical Gorenstein singularities, Mat. Sb. 196 (2005), no. 1, 81-122. MR 2141325

[Rei83] Miles Reid, Minimal models of canonical 3-folds, Algebraic varieties and analytic varieties (Tokyo, 1981), Adv. Stud. Pure Math., vol. 1, North-Holland, Amsterdam, 1983, pp. 131-180. MR 715649

[Rei94] , Nonnormal del Pezzo surfaces, Publ. Res. Inst. Math. Sci. 30 (1994), no. 5, 695-727. MR MR1311389 (96a:14042)

[Siu91] Yum Tong Siu, Some problems of rigidity in several complex variables, International Symposium in Memory of Hua Loo Keng, Vol. II (Beijing, 1988), Springer, Berlin, 1991, pp. 265-284. MR 1135842 
[Wil92] P. M. H. Wilson, The Kähler cone on Calabi-Yau threefolds, Invent. Math. 107 (1992), no. 3, 561-583. MR 1150602

[Wil93] Erratum: "The Kähler cone on Calabi-Yau threefolds" [Invent. Math. 107 (1992), no. 3, 561-583; MR1150602 (93a:14037)], Invent. Math. 114 (1993), no. 1, 231-233. MR 1235026

[Wil94] Minimal models of Calabi-Yau threefolds, Classification of algebraic varieties (L'Aquila, 1992), Contemp. Math., vol. 162, Amer. Math. Soc., Providence, RI, 1994, pp. 403-410. MR 1272711

[Wil97] _ Symplectic deformations of Calabi-Yau threefolds, J. Differential Geom. 45 (1997), no. 3, 611-637. MR 1472891

[Wil99] Flops, Type III contractions and Gromov-Witten invariants on Calabi-Yau threefolds, New trends in algebraic geometry (Warwick, 1996), London Math. Soc. Lecture Note Ser., vol. 264, Cambridge Univ. Press, Cambridge, 1999, pp. 465-484. MR 1714834

Thomas Peternell, Mathematisches Institut, Universität Bayreuth, 95440 Bayreuth, Germany

E-mail address: thomas.peternell@uni-bayreuth.de 